\title{
2008/55
}

Towards an understanding of tradeoffs between regional wealth, tightness of a common environmental constraint and the sharing rules

Raouf Boucekkine, Jacek B. Krawczyk and Th. Vallée 
CORE

Voie du Roman Pays 34

B-1348 Louvain-la-Neuve, Belgium.

Tel (32 10) 474304

Fax (32 10) 474301

E-mail: corestat-library@uclouvain.be http://www.uclouvain.be/en-44508.html 


\title{
CORE DISCUSSION PAPER
}

$2008 / 55$

\section{Towards an understanding of tradeoffs between regional wealth, tightness of a common environmental constraint and the sharing rules}

\author{
Raouf BOUCEKKINE ${ }^{1}$, Jacek B. KRAWCZYK ${ }^{2}$ \\ and Thomas VALLEE ${ }^{3}$
}

October 2008

\begin{abstract}
Consider a country with two regions that have developed differently so that their current levels of energy efficiency differ. Each region's production involves the emission of pollutants, on which a regulator might impose restrictions. The restrictions can be related to pollution standards that the regulator perceives as binding the whole country (e.g., enforced by international agreements like the Kyoto Protocol). We observe that the pollution standards define a common constraint upon the joint strategy space of the regions. We propose a game theoretic model with a coupled constraints equilibrium as a solution to the regulator's problem of avoiding excessive pollution. The regulator can direct the regions to implement the solution by using a political pressure, or compel them to employ it by using the coupled constraints' Lagrange multipliers as taxation coefficients. We specify a stylised model that possesses those characteristics, of the Belgian regions of Flanders and Wallonia. We analytically and numerically analyse the equilibrium regional production levels as a function of the pollution standards and of the sharing rules for the satisfaction of the constraint. For the computational results, we use NIRA, which is a piece of software designed to min-maximise the associated Nikaido-Isoda function.
\end{abstract}

Keywords: coupled constraints, generalised Nash equilibrium, Nikaido-Isoda function, regional economics, environmental regulations.

JEL Classification: C6, C7, D7

\footnotetext{
${ }^{1}$ Department of Economics and CORE, Université catholique de Louvain, Belgium; and University of Glasgow, Scotland. E-mail: raouf.boucekkine@uclouvain.be. This author is also member of ECORE, the newly created association between CORE and ECARES.

2 Victoria University of Wellington, New Zealand.

${ }^{3}$ LEM, IEMN-IAE, Université de Nantes, France.
}

This paper presents research results of the Belgian Program on Interuniversity Poles of Attraction initiated by the Belgian State, Prime Minister's Office, Science Policy Programming. The scientific responsibility is assumed by the authors. 



\section{Introduction}

The aim of this paper ${ }^{1}$ is to examine the impact of imposition of emission constraints on regional revenues and the national revenue of a two-region economy. The regions that are somewhat controllable by a sole regulator will be composed of industries of diverging specialisations. We will study the revenues as functions of the sharing rules, adoptable by a regulator, for spreading the burden of the constraints' satisfaction among the regions.

The need for regulation might result from the regulator's wish to comply with a national emissions' quota assigned to the country through an international agreement (like the Kyoto Protocol). The split of the quota among the regions is always a contentious issue in the context of dissimilar profiles of the regional industries. Moreover, the split will be a highly controversial matter if the regions are ethnically different.

An example of such a problem is a disagreement between Wallonia and Flanders (two Belgian regions) regarding sharing the pollution cleaning burden, recently studied in [3] and [8]. In those papers, an impact of "grandfathering" emission permits on regional revenues in a small open multi-sector (multi-regional) economic model (Heckscher-Ohlin type) is considered. Other multi-ethnic countries (like Canada, UK or Switzerland) might be facing similar problems.

In this paper, we treat the industries, or regions, as competitive agents and analyse the resulting equilibrium policies as well as the corresponding outputs and payoffs, as a consequence of adoption of a sharing rule, for apportioning a pollution quota to each region. What makes our paper essentially different from the above cited publications is that we allow for an emission constraint upon the agents' joint strategy space. Assuming the presence of an industry-independent regulator, we then vary the levels of the agents' responsibility for the coupled constraint's satisfaction and suggest which sharing rules might be preferred by the regulator.

The problem's setup in this paper is conceptually similar to that of [9], [10], [15], [4], [12], [18], [7] and also [5]. The common feature is that all those papers deal with coupled constraints games, in which competitive agents maximise their utility functions subject to constraints upon their joint strategy space. However, in this paper, we make explicit the relationship between a solution to the problem and the weights, which the regulator may use to distribute the responsibility for satisfaction of a joint constraint, among the agents. In that, we follow the seminal work [20] and use a coupled constraints equilibrium as a solution concept for the discussed problem. Under this solution concept the regulator can compute (for sufficiently concave games) the agents' strategies that are both unilaterally nonimprovable (Nash) and such that the constraints imposed on the joint strategy space are satisfied.

If the regulator can modify the agents' utilities and impose penalties for violation of the joint constraints then the game will become "decoupled" and the agents will implement the coupled constraints equilibrium in its "own interest", to avoid fines associated with excessive pollution. These penalties, which prevent excessive

\footnotetext{
${ }^{1}$ An earlier version of this paper was presented at 14 th International Conference on Computing in Economics and Finance, June 26-28, 2008, Sorbonne, Paris.
} 
pollution, can be computed using the coupled constraints Lagrange multipliers. However, for this modification of the players' utilities to induce the required behaviour, a coupled constraints equilibrium needs to exist and be unique for a given distribution of the responsibilities for the joint constraints satisfaction, among the agents. We will prove that our model possesses the property of diagonal strict concavity (DSC), which will be sufficient for uniqueness of a coupled constraints equilibrium. Obviously, the game has to possess the same properties should the sharing rules be implemented through a political process rather by threatening the regions with penalties .

Reports on that the energy more-intensive sector's revenue is proportionally more affected by the environmental policy than that of its less-intensive counterpart are provided by [3] and [8]. Our model's results suggest that the decision on apportioning a higher or lower energy share to a region should depend on an analysis of externalities, which the regions exert on each other. We also report on the various degrees of market "distortion" as a consequence of the imposition of pollution quotas and of the alteration of the rules for sharing the burden of the joint constraints' satisfaction. We expect our model can help the regulator discover which rules imply an acceptable degree of market distortion.

For the results we use NIRA, which is a piece of software designed to minmaximise the Nikaido-Isoda function and thus compute a coupled constraints equilibrium (see $[2,16]$ ). We also notice that a coupled constraints equilibrium could be obtained $^{2}$ as a solution to a quasi-variational inequality (see [11], [18]) or as a result of gradient pseudo-norm minimisation (see [20], [9], [10]).

What follows is a brief outline of what this paper contains. In Section 2 a stylised model of a two-region country is presented. Section 3 briefly explains the idea of a coupled constraints equilibrium and the algorithm that will be used to compute it. We present the calibrated model for a two-region environmental game in Section 5 and report on the equilibrium solutions. The concluding remarks summarise our findings, which include the economic interpretation of the results.

\section{A two-region country model}

\subsection{A game with constraints upon the agents' joint strat- egy space}

We understand a country as an entity, on whose territory Gross National Product (GNP) is generated. In some countries that have historically developed into regions, notwithstanding the inter-regional spill-over effects, the product created by one region's industry can be deemed somehow independent of the product created by the other region's industry. However, due to trade and, recently, environmental concerns, the supply of some production factors might be jointly constrained.

As a plausible application area for our analysis, we have mentioned in the Introduction the Belgian regions Flanders and Wallonia whose industries have developed distinctly over the last two centuries. In result, their contributions to the Belgian GNP and the overall "Belgian" pollution are significantly different.

\footnotetext{
${ }^{2}$ We refer to [13] for a review on numerical solutions to coupled-constraint equilibria.
} 
There is some evidence (see Table ${ }^{3} \mathrm{I}$ ) on that Flanders produces about $60 \%$ of GNP using about $60 \%$ of energy consumed by Belgium. On the other hand, Wallonia contributes to the gross product in about $24 \%$ but "burns" $33 \%$ of the country's energy supply. The remainders of GNP and energy consumption are the contributions by Brussels. However, we will not consider Brussels an active player in the game we define below. We believe that, firstly, Wallonia and Flanders see each other as direct competitors and neither of them "cares" much about what the regulator decides about the Brussels' quota. Secondly, Brussels' energy consumption appears small and, perhaps, no quota will make a big difference to its contribution to GNP.

Table I: Estimations of energy consumption and gross product generation in Belgium, about 2000

\begin{tabular}{|c||c|c||c|c||r|}
\hline \multicolumn{1}{|c||}{} & \multicolumn{1}{c||}{ Energy Use } & \multicolumn{2}{c||}{ Gross Value Added } & GVA/En.Use \\
\hline & $10^{6}$ boe & $\%$ & $10^{6}$ Euros & $\%$ & Euro/boe \\
\hline \hline Belgium & 270.065 & 100 & 223812.0 & 100 & 828.73 \\
\hline Brussels & 14.427 & 5.4 & 42562.5 & 19 & 2950.20 \\
\hline Wallonia & 89.832 & 33.3 & 52819.1 & 24 & 587.98 \\
\hline Flanders & 165.804 & 61.4 & 128146.6 & 57 & 772.88 \\
\hline
\end{tabular}

In Table I, we observe that Wallonia's usage of energy appears less effective than Flanders' is.

Intuitively, it seems possible to keep the overall pollution constant but vary its regional contributions and achieve an improvement of the whole country's performance. For example, using different sharing rules of apportioning the energy use between the two regions can force them use different equilibrium strategies, which may be more efficient from the social planner's point of view. It is the aim of the analysis conducted in this paper to help the "planners" to improve the latter.

Unless Wallonia's product is "badly" needed by Flanders, it may appear that encouraging Wallonia to use less energy (especially, if constrained) and allowing Flanders to use more of it, might be beneficial for the global revenue.

\footnotetext{
${ }^{3}$ The statistical data cited in this paper come from Belgostat [1] and [6]. The energy consumption is expressed in the table in boe. A barrel of oil equivalent (boe) is a unit of energy based on the approximate energy released by burning one barrel of crude oil. One boe contains approximately 0.143 toe (a ton of oil equivalent) or $6.1178632 \cdot 10^{9} \mathrm{~J}$ or about $1.70 \mathrm{MWh}$. Using boe rather than any other energy unit has the advantage that the price of 1 boe is the price OPEC charges their clients.
} 
Table II: Estimations of energy consumption and gross product generation per unit of labour in Belgium, about 2000

\begin{tabular}{|c||c|c|c|c|}
\hline & Employment & $\begin{array}{c}\text { Energy Use } \\
\text { /Employment }\end{array}$ & $\begin{array}{c}\text { Gross Value Added } \\
\text { /Employment }\end{array}$ & $\begin{array}{c}\text { GVA/Empl. } \\
/ \text { En. Use }\end{array}$ \\
\hline & & boe & Euros & Euro/boe \\
\hline \hline Belgium & 4085677 & 66.10 & 54780 & 0.0002 \\
\hline Brussels & 640992 & 22.51 & 66401 & 0.0046 \\
\hline Wallonia & 1093076 & 82.18 & 48322 & 0.0005 \\
\hline Flanders & 2351609 & 70.51 & 54493 & 0.0003 \\
\hline
\end{tabular}

Table II presents a slightly different picture. Here, the regional performance is expressed in relation to the size of labour, employed in each region. We see that Wallonia's usage of energy per worker is the highest and the revenue generated by a unit of labour is the lowest. ${ }^{4}$ In the rest of this paper we will propose and analyse a few models to help politicians decide about the energy sharing rules that could lead to a socially preferred equilibrium. ${ }^{5}$

\subsection{Model specification}

In this paper we consider a country of two regional industries $i=w, f$ (we will use for $w=$ Wallonia and for $f=$ Flanders) that generate output according to a two-factor production function. Let $e_{i}$ denote energy input per unit of labour in region $i$ where $^{6} e_{i} \in \mathbb{R}_{+} ; e \equiv\left[e_{i}, e_{-i}\right] \in \mathbb{R}_{+} \times \mathbb{R}_{+}$. One factor will be function $G_{i}\left(e_{i}\right)$ concave and smooth, dependent on energy used in sector $i$; the other factor $F_{i}\left(e_{i}, e_{-i}\right)$, also a concave and smooth function, dependent on energy used in the whole country, will represent the effects of learning-by-doing, knowledge spill-overs, externalities' impact, interregional flows, etc.. Energy will be purchased by each sector at international price $p$.

If the regions are separated geographically (or constitute different political or ethnic "units") their decisions about the use of energy, feeding into the factors $G_{i}(\cdot), F_{i}(\cdot, \cdot)$, can be regarded as independent of one another. If, for various reasons, each region strives to maximise its Gross Regional Revenue (GRR) we should look for each region's input and output levels as results of an equilibrium solution to a non-cooperative two-agent (or, two-region) game. If, in addition, the amount of energy to be used by the entire country is restricted ${ }^{7}$, a constraint needs to be added to the agents' joint strategy space. In result, the input and output will be

\footnotetext{
${ }^{4}$ The last column of Table II provides a rather complex and non standard measure of "efficiency", which is the revenue (in Euros) obtained out of one boe by one unit of labour. Here, Wallonia may appear more "economic" than the rest of the country in such per unit of labour terms. However, given the existing employment levels, it is impossible to claim on this basis that allowing Wallonia to use more energy would increase the whole country's revenue.

${ }^{5}$ As said in the Introduction, we are skeptical about the social planner's ability to enforce a Pareto efficient solution.

${ }^{6}$ Notation $-i$ signifies the other player.

${ }^{7}$ E.g., implied by a trade balance or the Kyoto Protocol.
} 
determined as a coupled constraints equilibrium, see $[20]^{8}$.

We assume there is one identical good generated by each region and this good's price is normalised to 1 . Below we propose a model for regional revenue $\Pi_{i}$, or the value added by region $i$ that directly depends on this good's production. The two values sum up to the national revenue.

Each regional revenue (or net value-added) $\Pi_{i} i=w, f$ will be expressed in monetary units and modelled as the difference between the "gross" good's value, modelled as product $F_{i}(\cdot, \cdot) G_{i}(\cdot)$ (multiplied by price=1), and the cost of energy input $e_{i}$ as follows:

$$
\Pi_{i}(e)=\underbrace{F_{i}\left(e_{i}, e_{-i}\right)}_{\text {spill-overs, etc. }} G_{i}\left(e_{i}\right)-p e_{i}
$$

One could consider several realisations of $G_{i}(\cdot)$ and $F_{i}(\cdot, \cdot)$. For example,

a. $F_{i}\left(e_{i}, e_{-i}\right) \equiv$ constant (no spill-overs, no externalities). This model would correspond to autarkic development of the regions; however it could also serve as a benchmark case, to isolate the elementary economic mechanisms of regional competition.

b. $F_{i}\left(e_{i}, e_{-i}\right)=\left(e_{-i}\right)^{\delta_{i}}$ (strategic complementarities). This would be a simple formulation of strategic complementarities: region $-i$ exerts an externality on the other region. If $\delta_{i}>0$ then the externality is positive.

c. $F\left(e_{i}, e_{-i}\right)=\left(e_{i}+e_{-i}\right)^{\delta_{i}}$ (production spill-overs $\left.{ }^{9}\right)$. In essence the production of one region depends also of the total energy used by the country.

d. $G\left(e_{i}\right)=\alpha_{i} e_{i}^{\beta_{i}}$. Coefficient $\alpha_{i}$ is total factor productivity. If $F_{i}(\cdot)$ is like in (b.), $0<\beta_{i}, 0<\delta_{i}$ and $\beta_{i}+\delta_{i}<1$ then the first term ("output") of $\Pi_{i}(e)$ is a Cobb-Douglas production function with diminishing returns to scale.

e. $G\left(e_{i}\right)=\alpha_{i} \ln \left(e_{i}\right)$. To avoid $\ln \left(e_{i}\right)<0$ we shall scale the model so that $e_{i}$ will always check $e_{i}>1$, see Section 4 . In conjunction with $F_{i}\left(e_{i}, e_{-i}\right)=$ constant (see (a.)), the corresponding $\Pi_{i}(e)$ and $\Pi_{-i}(e)$ constitute (arguably) the simplest pair of the revenue functions that retain the production-function (strict) concavity feature.

Notice that (1) and the factor realisations (a.)-(e.) capture several basic facts (albeit with a different degree of accuracy) about regional economics that a game model should encapsulate. In particular, expression (1) says it is costly to use energy and all choices (a.)-(e.) reflect the fact that using energy increases output. On the other hand, in each realisation we have abstracted from labour. We assume that the variables are expressed in per-unit-of-labour terms.

We can summarise some features of the above choices as follows:

\footnotetext{
${ }^{8}$ Or see [9], [10], [15], [4], [12], [5].

${ }^{9}$ In this realisation, total factor productivity $F\left(e_{i}, e_{-i}\right)$ is the same in both regions. This is so because it captures total non-appropriable knowledge derived from all productive activities in the economy, here proxied by the associated energy inputs. This specification is in the spirit of [19].
} 
- if $\alpha_{i}>\alpha_{-i}$ then region $i$ 's total factor productivity is higher than region $-i$ 's; this might suggest technology in region $i$ is more energy efficient than that of region $-i$;

- if $\beta_{i}>\beta_{-i}$ then region $i$ 's output is more elastic to energy changes than region $-i$ 's;

- if $\delta_{i}>\delta_{-i}$ than the externality produced by region $-i$ is more important for production of region $i$ than the other way around.

To highlight the main features of energy usage rationalisation we will use a benchmark model that combines (a.) and (e.)

$$
\Pi_{i}(e)=\alpha_{i} \ln \left(e_{i}\right)-p e_{i}
$$

This simple model will also help us to motivate the need for a numerical analysis of a more complicated model. It will be the combination of (c.) and (d.) as follows

$$
\Pi_{i}(e)=\alpha_{i}\left(e_{-i}\right)^{\delta_{i}}\left(e_{i}\right)^{\beta_{i}}-p e_{i}
$$

As said before, this choice captures the likely fact that region $-i$ (the "other" region) produces positive externality that feeds into the production of region $i$.

Let $E_{i}$ denote the amount of energy used in region $i, i=w, f$. As $e_{i}$ the amount of energy used by a unit of labour then

$$
E_{i}=\eta_{i} e_{i}
$$

where $\eta_{i}>0$ is the quantity ${ }^{10}$ of labour in region $i$.

Believing the sectors are "burning" (predominantly) oil to obtain energy, the emissions $M_{i}$ can be assumed a linear function of energy $E_{i}$ as follows

$$
M_{i}=\kappa_{i} E_{i}, \quad \kappa_{i}>0
$$

where $\kappa_{i}$ characterises the "burning" technology of region $i$.

In case the whole country is striving to curb its emissions below $M>0$ (where $M$ could result from the Kyoto protocol) the maximisation of (1) (or (2) or (3)) needs to allow for the following constraint

$$
M_{i}+M_{-i} \leq M \quad \Rightarrow \quad \kappa_{i} E_{i}+\kappa_{-i} E_{-i} \leq M
$$

If the emissions $M_{i}$ generated by a unit of $E_{i}$ were identical in each industry then $\kappa_{i}=\kappa_{-i}=\kappa$ and the constraint (6) could be rewritten as

$$
\eta_{i} e_{i}+\eta_{-i} e_{-i} \leq E
$$

which is imposed upon the joint strategy space $\mathbb{R}_{+} \times \mathbb{R}_{+} \ni\left[e_{i}, e_{-i}\right]$ and where $E=\frac{M}{\kappa}$ is the energy available to the whole country.

\footnotetext{
${ }^{10}$ Variable $e_{i}$ can be expressed in per-worker units, per hour, per worker-hour, etc.. The quantity $\eta_{i}$ will correspond to the measure of $e_{i}$.
} 
Given our assumption that the regions are "playing" a non-cooperative game, the optimal energy usage levels and the regional products can be obtained as a solution to a coupled constraint game defined as follows:

$$
\left.\begin{array}{rll}
\Pi_{i}\left(e^{*}\right)= & \max & \Pi_{i}\left(e_{i}, e_{-i}^{*}\right) \\
e_{i} & \text { s.t. } & \eta_{i} e_{i}+\eta_{-i} e_{-i}^{*} \leq E \quad i=w, f
\end{array}\right\}
$$

where $e^{*}=\left[e_{i}^{*}, e_{-i}^{*}\right]$ and we wrote $\Pi_{i}\left(e_{i}, e_{-i}^{*}\right)$ to stress that player $i$ needs to allow for the optimal action of player $-i$, in deciding about her own optimal level $e_{i}^{*}$.

A solution to (8) is such that no region can improve its own payoff by a unilateral action without breaching (7). Hence, this solution is a "generalised" Nash-Cournot equilibrium as it is called in e.g., [18], or a coupled constraint equilibrium as we call

it. If it exists it depends on a vector of weights $r_{i},(i=w, f)$ that can be viewed as a political instrument, which the central government can use to distribute the burden of satisfaction of the coupled constraint (7), among the players (regions or industries). We explain this concept in section 3.

\section{Constrained equilibria}

\subsection{Coupled constraints equilibria}

An equilibrium defined by (8) is a coupled constraint equilibrium.

A coupled constraints equilibrium (CCE) is an extension of a standard Nash equilibrium in which players' strategy sets are allowed to depend upon other players' strategies. Coupled constraints equilibria are also known as generalised Nash equilibria. The competition between the regions subject to the energy constraint described as is an example of such a problem. Analytical solutions to CCE problems are not normally possible so section A describes a numerical method for solving some such problems.

Coupled constraints equilibria are particularly useful in a class of problems where competing agents are subjected to regulation. Many electricity market and environmental problems belong to this class see e.g., [9], [10], [4] where this concept has been applied in microeconomic contexts; see [7] for an international economics application. In general, CCE allows modelling of a situation in which the actions of one player condition how 'big' the actions of other players can be. Constraints in which the actions of one player do not affect the action space of another (as in Nash equilibrium problems) are called uncoupled.

In these games the constraints are assumed to be such that the resulting collective action set $X$ is a closed convex subset of $\mathbb{R}_{m}$. If $X_{i}$ is player- $f$ 's action set, $X \subseteq X_{1} \times \cdots \times X_{F}$ is the collective action set (where $X=X_{1} \times \cdots \times X_{F}$ represents the special case in which the constraints are uncoupled).

Allowing for the above, a solution to (8) can be explained as follows. Let the collective action $\mathrm{x}^{*}$ be the game solution and the players' payoff functions, $\Pi_{i}$, be continuous in all players' actions and concave in their own action. The Nash equilibrium can be written as

$$
\Pi_{i}\left(\mathbf{x}^{*}\right)=\max _{\mathbf{x} \in X} \Pi_{i}\left(y_{i} \mid \mathbf{x}^{*}\right)
$$


where $y_{f} \mid \mathbf{x}^{*} \in X$ denotes a collection of actions where the $f$ th agent "tries" $y_{i}$ while the remaining agents continue to play the collective action $\mathbf{x}^{*}$. Note that $\mathbf{x}^{*}$ is a column vector with elements $x_{g}, g=1,2, \ldots, f-1, f+1, \ldots, F$. At $\mathbf{x}^{*}$ no player can improve his own payoff through a unilateral change in his strategy so $\mathbf{x}^{*}$ is a Nash equilibrium point. If $X$ is a closed and strictly convex set defined through coupled constraints (like (7)) then $\mathbf{x}^{*}$ is a CCE.

Games with coupled constraints rarely allow for an analytical solution and so numerical methods must be employed. In this paper, we will solve game (8), with the revenue functions defined by (3), using a method based on the Nikaido-Isoda function and a relaxation algorithm (hence the name: NIRA), explained in Appendix A.

\subsection{Existence and uniqueness of equilibrium points}

It is one thing to know that one has a method to solve games with constraints but, before proceeding, one needs to establish that the game has an equilibrium at all. Furthermore, since the NIRA algorithm converges to a single equilibrium point it would be nice if that equilibrium could be shown to be unique. The conditions for existence and uniqueness for games with coupled constraints will be given in Section 3.2.1. The equivalent definition that relies upon the notion weak convexconcavity of the Nikaido-Isoda function, used for the numerical solutions in this paper, is formulated in Section 3.2.2. In particular, the satisfaction of Condition (e.) of Theorem 3.2 (formulated in Section 3.2.2, see page 10) implies diagonal strict concavity (explained in Section 3.2.1 in page 9) of a game whose NikadoIsoda function is weakly convex-concave (see [21], Theorem 5.2.1). Hence any game which satisfies the convergence conditions for the relaxation algorithm will satisfy the diagonal strict concavity condition required for the unique solution to the Rosen coupled constraint game.

\subsubsection{Diagonal strict concavity}

We know from [20] ${ }^{11}$ that an equilibrium exists and is unique if the game is $d i$ agonally strictly concave. We summarise (after [14]) the main ingredients needed for the confirmation that the constrained game (8) has a unique equilibrium. We formulate theorem 3.1 for a two-player case as considered in this paper ${ }^{12}$.

Denote $e \equiv\left[e_{w}, e_{f}\right], \rho(e, r), r \in \mathbb{R}^{2}, r_{i}>0, \forall i$ the "combined payoff" (or joint payoff function) as

$$
\rho(e, r)=r_{w} \Pi_{w}(e)+r_{f} \Pi_{f}(e),
$$

and $g(e, r)$ the pseudo-gradient of $\rho(e, r)$,

$$
g(e, r)=\left[\begin{array}{c}
r_{w} \frac{\partial \Pi_{w}(e)}{\partial e_{w}} \\
r_{f} \frac{\partial \Pi_{f}(e)}{\partial x_{f}}
\end{array}\right] .
$$

\footnotetext{
${ }^{11}$ Also see [14] or [13] for some applications.

${ }^{12}$ The original Rosen theorem in [20] is valid for $n$ players.
} 
Definition 3.1. The function $\rho(e, r)$ will be called diagonally strictly concave in $e$, if for every $e=\left(e_{w}, e_{f}\right)^{T}$ and $e^{\prime}=\left(e_{w}^{\prime}, e_{f}^{\prime}\right)^{T}, e_{w}+e_{f} \leq E$ and fixed $r \in \mathbb{R}_{+}^{2}$, we have

$$
\left(e-e^{\prime}\right)^{T} g\left(e^{\prime}, r\right)+\left(e^{\prime}-e\right)^{T} g(e, r)>0,
$$

where $^{T}$ means transposition.

We often call a game diagonally strictly concave whose joint payoff function is diagonally strictly concave.

Lemma 3.1. If $\Pi_{i}(e), i=w, f$ are "enough" differentiable, a sufficient condition that $\rho(e, r)$ be diagonally strictly concave in e for fixed $r>0$ is that the "pseudoHessian" symmetric matrix

$$
\mathcal{H}=G(e, r)+G^{T}(e, r)
$$

be negative definite for $e \in S$. Here the matrix $G(e, r)$ is the Jacobian with respect to $e$ of gradient $g(e, r)$.

Theorem 3.1. If $\rho(e, r)$ is diagonally strictly concave for some $r \in \mathbb{R}_{+}^{2} \backslash\{0\}$, then the Nash equilibrium point of game (8) exists and is unique.

We will apply this theorem to games considered in Sections 4 and 5 .

\subsubsection{Convergence theorem}

A weakly convex-concave function is a bivariate function that exhibits weak convexity in its first argument and weak concavity in its second argument. The next three definitions (see [17] or [22]) formalise this notion. ${ }^{13}$ As Theorem 3.2 (the convergence theorem) will document, weak convex-concavity of a function is a crucial assumption needed for convergence of a relaxation algorithm to a coupled constraints equilibrium.

Let $X$ be a convex closed subset of the Euclidean space $\mathbb{R}^{m}$ and $f$ a continuous function $f: X \rightarrow \mathbb{R}$.

Definition 3.2. A function of one argument $f(\mathbf{x})$ is weakly convex on $X$ if there exists a function $r(\mathbf{x}, \mathbf{y})$ such that $\forall \mathbf{x}, \mathbf{y} \in X$

$$
\begin{aligned}
& \alpha f(\mathbf{x})+(1-\alpha) f(\mathbf{y}) \geq f(\alpha \mathbf{x}+(1-\alpha) \mathbf{y})+\alpha(1-\alpha) r(\mathbf{x}, \mathbf{y}) \\
& 0 \leq \alpha \leq 1, \text { and } \frac{r(\mathbf{x}, \mathbf{y})}{\|\mathbf{x}-\mathbf{y}\|} \rightarrow 0 \text { as }\|\mathbf{x}-\mathbf{y}\| \rightarrow 0 \quad \forall \mathbf{x} \in X .
\end{aligned}
$$

Definition 3.3. A function of one argument $f(\mathbf{x})$ is weakly concave on $X$ if there exists a function $\mu(\mathbf{x}, \mathbf{y})$ such that, $\forall \mathbf{x}, \mathbf{y} \in X$

$$
\begin{gathered}
\alpha f(\mathbf{x})+(1-\alpha) f(\mathbf{y}) \leq f(\alpha \mathbf{x}+(1-\alpha) \mathbf{y})+\alpha(1-\alpha) \mu(\mathbf{x}, \mathbf{y}) \\
0 \leq \alpha \leq 1, \text { and } \frac{\mu(\mathbf{x}, \mathbf{y})}{\|\mathbf{x}-\mathbf{y}\|} \rightarrow 0 \quad \text { as } \quad\|\mathbf{x}-\mathbf{y}\| \rightarrow 0 \quad \forall \mathbf{x} \in X .
\end{gathered}
$$

\footnotetext{
${ }^{13}$ Recall the following elementary definition: a function is "just" convex $\Longleftrightarrow$

$$
\alpha f(\mathbf{x})+(1-\alpha) f(\mathbf{y}) \geq f(\alpha \mathbf{x}+(1-\alpha) \mathbf{y}), \quad \alpha \in[0,1] .
$$
}


Example: The convex function $f(x)=x^{2}$ is weakly concave (see [15]) but the convex $\overline{\text { function }} f(x)=|x|$ is not.

Now take a bivariate function $\Psi: X \times X \rightarrow \mathbb{R}$ defined on a product $X \times X$, where $X$ is a convex closed subset of the Euclidean space $\mathbb{R}^{m}$.

Definition 3.4. A function of two vector arguments, $\Psi(\mathbf{x}, \mathbf{y})$ is referred to as weakly convex-concave if it satisfies weak convexity with respect to its first argument and weak concavity with respect to its second argument.

The functions $r(\mathbf{x}, \mathbf{y} ; \mathbf{z})$ and $\mu(\mathbf{x}, \mathbf{y} ; \mathbf{z})$ were introduced with the concept of weak convex-concavity and are called the residual terms. Notice that smoothness of $\Psi(\mathbf{z}, \mathbf{y})$ is not required. However, if $\Psi(\mathbf{x}, \mathbf{y})$ is twice continuously differentiable with respect to both arguments on $X \times X$, the residual terms satisfy (see [15])

$$
r(\mathbf{x}, \mathbf{y} ; \mathbf{y})=\frac{1}{2}\langle A(\mathbf{x}, \mathbf{x})(\mathbf{x}-\mathbf{y}), \mathbf{x}-\mathbf{y}\rangle+o_{1}\left(\|\mathbf{x}-\mathbf{y}\|^{2}\right)
$$

and

$$
\mu(\mathbf{y}, \mathbf{x} ; \mathbf{x})=\frac{1}{2}\langle B(\mathbf{x}, \mathbf{x})(\mathbf{x}-\mathbf{y}), \mathbf{x}-\mathbf{y}\rangle+o_{2}\left(\|\mathbf{x}-\mathbf{y}\|^{2}\right)
$$

where $A(\mathbf{x}, \mathbf{x})=\left.\Psi_{\mathbf{x x}}(\mathbf{x}, \mathbf{y})\right|_{\mathbf{y}=\mathbf{x}}$ is the Hessian of the Nikaido-Isoda function with respect to the first argument and $B(\mathbf{x}, \mathbf{x})=\left.\Psi_{\mathbf{y y}}(\mathbf{x}, \mathbf{y})\right|_{\mathbf{y}=\mathbf{x}}$ is the Hessian of the Nikaido-Isoda function with respect to the second argument, both evaluated at $\mathbf{y}=\mathbf{x}$.

To prove the inequality of condition (e) of Theorem 3.2 (the convergence theorem, below) under the assumption that $\Psi(\mathbf{x}, \mathbf{y})$ is twice continuously differentiable, it suffices to show that

$$
Q(\mathbf{x}, \mathbf{x})=A(\mathbf{x}, \mathbf{x})-B(\mathbf{x}, \mathbf{x})
$$

is strictly positive definite.

Theorem 3.2 (Convergence theorem). There exists a unique normalised Nash equilibrium point to which the algorithm (50) converges if:

a. $X$ is a convex, compact subset of $\mathbb{R}^{m}$,

b. the Nikaido-Isoda function $\Psi: X \times X \rightarrow I R$ is a weakly convex-concave function and $\Psi(\mathbf{x}, \mathbf{x})=0$ for $\mathbf{x} \in X$,

c. the optimum response function $Z(\mathbf{x})$ is single valued and continuous on $X$,

d. the residual term $r(\mathbf{x}, \mathbf{y} ; \mathbf{z})$ is uniformly continuous on $X$ w.r.t. $\mathbf{z}$ for all $\mathbf{x}, \mathbf{y} \in X$,

e. the residual terms satisfy

$$
r(\mathbf{x}, \mathbf{y} ; \mathbf{y})-\mu(\mathbf{y}, \mathbf{x} ; \mathbf{x}) \geq \beta(\|\mathbf{x}-\mathbf{y}\|), \quad \mathbf{x}, \mathbf{y} \in X
$$

where $\beta(0)=0$ and $\beta$ is a strictly increasing function (i.e., $\beta\left(t_{2}\right)>\beta\left(t_{1}\right)$ if $\left.t_{2}>t_{1}\right)$, 
$f$. the relaxation parameters $\alpha_{s}$ satisfy

- either (non-optimised step)

(a) $\alpha_{s}>0$,

(b) $\sum_{s=0}^{\infty} \alpha_{s}=\infty$,

(c) $\alpha_{s} \rightarrow 0$ as $s \rightarrow \infty$.

- or (optimised step)

$$
\alpha_{s}=\arg \min _{\alpha \in[0,1)}\left\{\max _{\mathbf{y} \in X} \Psi\left(\mathbf{x}^{(s+1)}(\alpha), \mathbf{y}\right)\right\} .
$$

Proof. See [15] for a proof.

\subsection{Enforcement through taxation}

Once a CCE, $x^{*}$, has been computed it is possible to create an unconstrained game which has $x^{*}$ as its solution by a simple modification to the players' payoff functions. For example, a regulator may compute that $x^{*}$ is the $\mathrm{CCE}$ of a game involving the desired constraints on agents' behaviour. He may then wish to induce the players to arrive at this point through a scheme of taxation that modifies their payoff functions. This can be achieved by the use of penalty functions that punish players for breaching the coupled constraints.

Penalty functions are weighted by the Lagrange multipliers obtained from the constrained game. For each constraint, players are taxed according to the function

$$
T_{\ell, i}\left(\lambda, r_{i}, \mathbf{x}\right)=\frac{\lambda_{\ell}}{r_{i}} \max \left(0, Q_{\ell}(\mathbf{x})-\bar{Q}_{\ell}\right)
$$

where $\lambda_{\ell}$ is the Lagrange multiplier associated with the $\ell$ th constraint and $Q_{\ell}(\mathbf{x})$ can be the amount of energy as described by the left hand side of (7). Symbol $\bar{Q}_{\ell}, \ell=1,2, \ldots L$ denotes the corresponding limits ( $L$ is the total number of constraints $\left.{ }^{14}\right) ; \mathrm{x}$ is the vector of players' actions, $r_{i}$ is player $f$ 's weight that defines their responsibility for the constraints' satisfaction.

If the weights $\mathbf{r}$ were identical $[1,1, \ldots 1]$ then the penalty term for constraint $\ell$ is the same for each player $f$

$$
T_{\ell, i}(\lambda, 1, \mathbf{x})=\lambda_{\ell} \max \left(0, Q_{\ell}(\mathbf{x})-\bar{Q}_{\ell}\right) .
$$

Hence, if the weight for player $f$ is for example $r_{i}>1$ and the weights for the other players were $1,1, \ldots 1$, then the responsibility of player $f$ for the constraints' satisfaction is lessened. Obviously, if the players' responsibilities are distributed in periods $t$ non-equally, then the weights would become $r_{i}^{t}$.

The players' payoff functions, so modified, will be

$$
\underline{\Pi}_{i}(\mathbf{x})=\Pi_{i}(\mathbf{x})-\sum_{\ell} T_{\ell i}(\lambda, \mathbf{r}, \mathbf{x}) .
$$

\footnotetext{
${ }^{14}$ Here, we have $\ell=L=1$.
} 
Notice that under this taxation scheme the penalties remain "nominal" (i.e., zero) if all constraints are satisfied.

The Nash equilibrium of the new unconstrained game with payoff functions $\bar{\Pi}$ is implicitly defined by the equation

$$
\underline{\Pi}\left(\mathbf{x}^{* *}\right)=\max _{y_{i} \in \mathbb{R}^{+}} \underline{\Pi}\left(y_{i} \mid \mathbf{x}^{* *}\right) \quad \forall i,
$$

(compare with equation (9)). For the setup of the problem considered in this paper $x^{*}=x^{* *}$. That is, the CCE is equal to the unconstrained equilibrium with penalty functions for breaches of the constraints, weighted by the Lagrange multipliers (see [15], [12] and [13] for a more detailed discussion).

\section{The benchmark problem}

\subsection{Existence and uniqueness of equilibrium}

We will formulate and analyse a simple problem of energy apportioning between two regions with no externalities and no spill-over effects. We will also see that studying the solutions to this "simplest" game of requires some computational analysis.

Consider two "autarkic" regions $i=w, f$ with the revenue functions based on (2) (see page 7) i.e.,

$$
\begin{aligned}
\Pi_{w}(e) & =\ln \left(e_{w}\right)-p e_{w} \\
\Pi_{f}(e) & =\alpha_{f} \ln \left(e_{f}\right)-p e_{f}
\end{aligned}
$$

where, for simplicity, we assumed $\alpha_{w}=1$. As said in Section 2.2, we will scale the model appropriately (see footnote 17) to avoid $e_{i} \leq 1, i=w, f$.

The regions face a joint constraint (compare (7))

$$
e_{w}+\eta_{f} e_{f} \leq E
$$

where, for simplicity, we assumed $\eta_{w}=1$. Naturally $\eta_{w} \geq 0, \eta_{f} \geq 0$.

To claim equilibrium existence and uniqueness (see Theorem 3.1) we need to prove Lemma 3.1. Matrix $\mathcal{H}$ (see (13)) for game (8) with the revenue functions $(25),(26)$ is

$$
\mathcal{H}_{1}=\left[\begin{array}{cc}
-\frac{r_{w}}{e_{w}{ }^{2}} & 0 \\
0 & -\frac{r_{f} \alpha_{f}}{e_{f}^{2}}
\end{array}\right] .
$$

Clearly $\mathcal{H}_{1}$ is strictly negative definite. The constraint set determined by (27) and $e_{w} \geq 0, e_{f} \geq 0$ is convex. Hence the game defined by payoffs (25), (26) is diagonally strictly convex. Consequently, if we fix the weights $\left(r_{w}, r_{f}\right) \in \mathbb{R}_{+}^{2}$ and compute an equilibrium then the equilibrium is unique. The other theorems' results, proved in [20] for diagonally strictly convex games, which will be referred to below, are applicable to this game. 
By definition, a coupled constraint equilibrium $\left(e_{w}^{*}, e_{f}^{*}, \lambda^{*}\right)$ is to be determined as the triple that satisfies:

$$
\begin{aligned}
& \left.\ln \left(e_{w}^{*}\right)-p e_{w}^{*} \geq \ln \left(e_{w}\right)-p e_{w}+\lambda_{w}\left(-e_{w}-\eta_{f} e_{f}^{*}+E\right)\right\} \\
& \left.\alpha_{f} \ln \left(e_{f}^{*}\right)-p e_{f}^{*} \geq \alpha_{f} \ln \left(e_{f}\right)-p e_{f}+\lambda_{f}\left(-e_{w}^{*}-\eta_{f} e_{f}+E\right)\right\}
\end{aligned}
$$

where $\lambda_{w} \geq 0, \lambda_{f} \geq 0$ and $\lambda_{w}\left(-e_{w}^{*}-\eta_{f} e_{f}^{*}+E\right), \lambda_{f}\left(-e_{w}^{*}-\eta_{f} e_{f}^{*}+E\right)$. From $[20]^{15}$ we know that that for every concave game there exists a (Rosen-)normalised equilibrium point $\left(e_{w}^{*}, e_{f}^{*}\right)$ with $\lambda_{w}^{*}=\frac{\lambda^{*}}{r_{w}}, \lambda_{f}^{*}=\frac{\lambda^{*}}{r_{f}}$ where $\lambda^{*}$ is a joint Lagrange multiplier ("shadow" price) that corresponds to constraint (27).

If so and by the necessity of the Kuhn-Tucker-Karush conditions, in equilibrium $\left(e_{w}^{*}, e_{f}^{*}, \lambda^{*}\right)$ satisfy

$$
\left.\begin{array}{rl}
-e_{w}^{*}-\eta_{f} e_{f}^{*}+E & 0 \\
\lambda^{*} \geq & 0 \\
\lambda^{*}\left(-e_{w}^{*}-\eta_{f} e_{f}^{*}+E\right) & =0 \\
\frac{1}{e_{w}^{*}}-p-\lambda^{*} & =0 \\
\frac{\alpha_{f}}{e_{f}^{*}}-p-\eta_{f} \frac{\lambda^{*}}{r_{f}} & =0
\end{array}\right\}
$$

where for simplicity we assumed $r_{w}=1 .^{16}$

\subsection{Properties of equilibrium}

Given $r_{f}$, the coupled constraint equilibrium for the interesting case of $\lambda^{*}>0$ is available albeit as a function of $\lambda^{*}$. The equilibrium energy usage ${ }^{17}$ per worker is

$$
e_{w}^{*}=\frac{1}{p+\lambda^{*}}, \quad e_{f}^{*}=\frac{\alpha_{f} r_{f}}{r_{f} p+\eta_{f} \lambda^{*}}=\frac{\alpha_{f}}{p+\frac{\eta_{f} \lambda^{*}}{r_{f}}} .
$$

However, these relations are functions of the equilibrium shadow price $\lambda^{*}$. (We notice that the marginal cost of violating the constraint is diminished $r_{f}$-times for the player whose $r_{i} \neq 1$.) To explicit the equilibrium shadow-price dependence on the problem parameters (including $r_{f} \neq 1$ ) one has to solve the following equation

$$
\frac{1}{p+\lambda^{*}}+\frac{\alpha_{f} \eta_{f}}{p+\frac{\eta_{f} \lambda^{*}}{r_{f}}}=E
$$

\footnotetext{
${ }^{15}$ See Theorem 3 in [20].

${ }^{16}$ Rosen [20] analyses equilibria for weights $r_{i} \in \mathbb{R}_{+}, i=1,2, \ldots N$. We prove in Appendix B, for $N=2$, equivalence between equilibria obtained for $\left[r_{w}, r_{f}\right] \in \mathbb{R}_{+}^{2}$, and when $r_{w}=1$ and $r_{f} \in(0, \infty)$.

${ }^{17}$ For $e_{i}^{*}>1$ so that we avoid negative output, $p+\lambda^{*}<1$. To assure this result we can scale the model as follows: choose the energy unit so that price $p<1$; then scale total factor productivity $\alpha_{f}$ (and/or the labour units) with respect to $E$ so that the ratios appearing in (30) are larger than 1 .
} 
which is a quadratic equation in $\lambda^{*}$

$$
\eta_{f} E\left(\lambda^{*}\right)^{2}-\left(\eta_{f}+\eta_{f} r_{f} \alpha_{f}-E p\left(\eta_{f}+r_{f}\right)\right) \lambda^{*}-p r_{f}\left(1+\eta_{f} \alpha_{f}-E p\right)=0 .
$$

The roots of this equation are

$$
\begin{aligned}
\lambda_{1}^{*} & =\frac{\eta_{f}\left(1+r_{f} \alpha_{f}\right)-E p\left(\eta_{f}+r_{f}\right)}{2 E \eta_{f}} \\
& +\frac{\sqrt{\left(\eta_{f}+\eta_{f} r_{f} \alpha_{f}\right)^{2}-2 E p \eta_{f}\left(\eta_{f}-r_{f}-\eta_{f} r_{f} \alpha_{f}+\alpha_{f} r_{f}^{2}\right)+E^{2} p^{2}\left(\eta_{f}-r_{f}\right)^{2}}}{2 E \eta_{f}} \\
\lambda_{2}^{*} & =\frac{\eta_{f}\left(1+r_{f} \alpha_{f}\right)-E p\left(\eta_{f}+r_{f}\right)}{2 E \eta_{f}} \\
& -\frac{\sqrt{\left(\eta_{f}+\eta_{f} r_{f} \alpha_{f}\right)^{2}-2 E p \eta_{f}\left(\eta_{f}-r_{f}-\eta_{f} r_{f} \alpha_{f}+\alpha_{f} r_{f}^{2}\right)+E^{2} p^{2}\left(\eta_{f}-r_{f}\right)^{2}}}{2 E \eta_{f}}
\end{aligned}
$$

Any exact conclusions about the relationship between $r_{f}$ and the positive root $\lambda^{*}$ are parameter specific and would require simulation. We will solve numerically a more realistic game in Section 5 to analyse this relationship. Here, however, we can formulate Proposition 4.1 to demonstrate a few simple general properties, which the shadow price $\lambda^{*}$ satisfies in coupled constraint equilibrium (described by (30) and $(31))$.

Proposition 4.1. In the unique coupled constraint equilibrium of the benchmark bi-regional game (25)-(27), the constraint's shadow price $\lambda^{*}$ possesses the following properties:

a. if the amount of available energy $E$ decreases, the shadow price $\lambda^{*}$ increases;

b. if the price of energy $p$ increases, then the shadow price $\lambda^{*}$ decreases;

c. if $r_{f}$ increases, which means a diminution of the responsibility of region $f$, $\lambda^{*}$ increases.

d. if labour supply $\eta_{f}$ or total factor productivity $\alpha_{f}$ increase, $\lambda^{*}$ increases. labelprop-b

The proofs are elementary and based on the analysis of (31) re-written as

$$
\frac{\alpha_{f} \eta_{f}}{p+\frac{\eta_{f} \lambda^{*}}{r_{f}}}=E-\frac{1}{p+\lambda^{*}} .
$$

For example, (a.) follows from the observation that if $\lambda^{*}$ decreased, rather than increased as is claimed in the proposition, then the right hand side of (34) would be negative. Similar reasoning proves the other items of the proposition. 
Remark 4.1. Notice that properties (a.)-(d.) are insufficient to claim that the privileged player's (here: region $f$ whose $r_{f}>1$ ) marginal cost of violating the constraint (see (21)) will always decrease for $r_{f}>1$. For the claim to be true, $\frac{d \lambda^{*}}{d r_{f}}<1$. We will examine a similar relationship in the game with externalities, solved numerically in the next section.

Equilibrium revenue per worker (as a function of $\lambda^{*}$ ) is

$$
\Pi_{w}^{*}=\ln \left(\frac{1}{p+\lambda^{*}}\right), \quad \Pi_{f}^{*}=\alpha_{f} \ln \left(\frac{\eta_{f} \alpha_{f}}{p+\frac{\eta_{f} \lambda^{*}}{r_{f}}}\right)
$$

and the country's (total) revenue

$$
\Pi_{w}^{*}+\Pi_{f}^{*}=\ln \left(\frac{1}{p+\lambda^{*}}\right)+\alpha_{f} \ln \left(\frac{\eta_{f} \alpha_{f}}{p+\frac{\eta_{f} \lambda^{*}}{r_{f}}}\right)=\ln \left(\frac{1}{p+\lambda^{*}}\right)\left(\frac{\eta_{f} \alpha_{f}}{p+\frac{\eta_{f} \lambda^{*}}{r_{f}}}\right)^{\alpha_{f}}
$$

The latter may grow in $r_{f}$ but only if the growth of $\lambda^{*}$ is slower than the rise of $r_{f}$, see Remark 4.1. We will analyse the issue of improvements of $\Pi_{w}^{*}+\Pi_{f}^{*}$ due to the changes in $r_{w}$ and $r_{f}$ numerically in Section 5 .

We also notice that, for this benchmark game without externalities or spill-over effects, the symmetric Pareto-optimal ("efficient") solution $\left(\bar{e}_{w}, \bar{e}_{f}, \bar{\lambda}\right) \geq 0$, which satisfies

$$
\ln \left(\bar{e}_{w}\right)-p \bar{e}_{w}+\ln \left(\bar{e}_{f}\right)-p \bar{e}_{f} \geq \ln \left(e_{w}\right)-p e_{w}+\ln \left(e_{f}\right)-p e_{f}+\bar{\lambda}\left(-e_{w}-\eta_{f} e_{f}+E\right)
$$

coincides $^{18}$ with $e_{w}^{*}, e_{f}^{*}, \lambda^{*}$ given in (30) and (31).

\section{A game with externalities}

\subsection{Uniqueness and equilibrium conditions}

Here we consider two competitive regions that face a joint constraint (compare (7))

$$
\eta_{w} e_{w}+\eta_{f} e_{f} \leq E
$$

and whose outputs are enhanced by positive externalities feeding into the opponents' revenue functions as in (3) (see page 7) i.e.,

$$
\Pi_{i}(e)=\alpha_{i}\left(e_{-i}\right)^{\delta_{i}}\left(e_{i}\right)^{\beta_{i}}-p e_{i}, \quad i=w, f .
$$

The pseudo-Hessian for game (8) with the revenue functions (39) is

\footnotetext{
${ }^{18}$ The symmetric Pareto-optimal solution is when the weights for each player's payoff are identical. Hence, we mean the coincidence with the game when $r_{f}=1$.
} 


$$
\begin{gathered}
\mathcal{H}_{2}= \\
{\left[\begin{array}{cc}
r_{w} \alpha_{w} e_{f}^{\delta_{w}} e_{w}{ }^{\beta_{w}-2} \beta_{w}\left(\beta_{w}-1\right) & \frac{r_{f} \alpha_{f} e_{w}{ }^{\delta_{f}} \delta_{f} e_{f}^{\beta_{f}} \beta_{f}+r_{w} \alpha_{w} e_{f}{ }^{\delta_{w}} \delta_{w} e_{w}{ }^{\beta_{w}} \beta_{w}}{2 e_{w} e_{f}} \\
\frac{r_{f} \alpha_{f} e_{w}{ }^{\delta_{f}} \delta_{f} e_{f}{ }^{\beta_{f}} \beta_{f}+r_{w} \alpha_{w} e_{f}^{\delta_{w}} \delta_{w} e_{w}{ }^{\beta_{w}} \beta_{w}}{2 e_{w} e_{f}} & r_{f} \alpha_{f} e_{w}{ }^{\delta_{f}} e_{f}{ }^{\beta_{f}-2} \beta_{f}\left(\beta_{f}-1\right)
\end{array}\right]}
\end{gathered}
$$

We can clearly see that $\mathcal{H}^{1,1}<0$. However, to determine the diagonal strict concavity of the game we also need $\operatorname{det} \mathcal{H}_{2}>0$. This result appears parameter dependent but will be satisfied at least for "large" $e_{w}$ and $e_{f}$. Notice that the product $\mathcal{H}^{1,2} \mathcal{H}^{2,1}$, to be subtracted from $\mathcal{H}^{1,1} \mathcal{H}^{2,2}>0$ (to compute the determinant) is vanishing for large $e_{w}$ and $e_{f}$; this is so because the powers of $e_{w}$ and $e_{f}$ in the denominator are greater than in the numerator.

We will check later in Section 5.3 (i.e., after calibration) that $\mathcal{H}_{2}$ is negative definite. Hence, if we fix the weights $r_{w}, r_{f}$ and compute an equilibrium for game (8) this equilibrium is unique.

As in Section 4, a coupled constraint equilibrium is the triple $\left(e_{w}^{*}, e_{f}^{*}, \lambda^{*}\right)$ that satisfies:

$$
\left.\begin{array}{rl}
\alpha_{w}\left(e_{f}^{*}\right)^{\delta_{w}}\left(e_{w}^{*}\right)^{\beta_{i}}-p e_{w}^{*} \geq \alpha_{w}\left(e_{f}^{*}\right)^{\delta_{w}}\left(e_{w}\right)^{\beta_{i}}-p e_{w}+\lambda_{w}\left(-\eta_{w} e_{w}-\eta_{f} e_{f}^{*}+E\right) \\
\alpha_{f}\left(e_{w}^{*}\right)^{\delta_{f}}\left(e_{f}^{*}\right)^{\beta_{i}}-p e_{f}^{*} \geq \alpha_{f}\left(e_{w}^{*}\right)^{\delta_{w}}\left(e_{f}\right)^{\beta_{i}}-p e_{f}+\lambda_{f}\left(-\eta_{w} e_{w}^{*}-\eta_{f} e_{f}+E\right)
\end{array}\right\}
$$

where $\lambda_{w} \geq 0, \lambda_{f} \geq 0$ and $\lambda_{w}\left(-e_{w}^{*}-\eta_{f} e_{f}^{*}+E\right), \lambda_{f}\left(-e_{w}^{*}-\eta_{f} e_{f}^{*}+E\right)$. As in Section 4, we invoke the results obtained in [20]. In particular we look for an equilibrium point $\left(e_{w}^{*}, e_{f}^{*}\right)$ with $\lambda_{w}^{*}=\frac{\lambda^{*}}{r_{w}}, \lambda_{f}^{*}=\frac{\lambda^{*}}{r_{f}}$ where $\lambda^{*}$ is a joint Lagrange multiplier ("shadow" price) that corresponds to constraint (38).

If so and by the necessity of the Kuhn-Tucker-Karush conditions, the triple $\left(e_{w}^{*}, e_{f}^{*}, \lambda^{*}\right)$ has to satisfy in equilibrium

$$
\left.\begin{array}{rl}
-\eta_{w} e_{w}^{*}-\eta_{f} e_{f}^{*}+E & \geq \\
\lambda^{*} & \geq 0 \\
\lambda^{*}\left(-\eta_{w} e_{w}^{*}-\eta_{f} e_{f}^{*}+E\right) & =0 \\
r_{w} \alpha_{w} e_{f}^{\delta_{w}} e_{w}^{\beta_{w}-1} \beta_{w}-r_{w} p-\eta_{w} \lambda^{*} & =0 \\
r_{f} \alpha_{f} e_{w}^{\delta_{f}} e_{f}{ }^{\beta_{f}-1} \beta_{f}-r_{f} p-\eta_{f} \lambda^{*} & =0
\end{array}\right\} .
$$

We can express the equilibrium strategies $\left(e_{w}^{*}, e_{f}^{*}\right)$ as functions of $\lambda^{*}$ for the interesting case of $\lambda^{*}>0$. However, after the substitution of the strategies in the energy balance condition $\left(\eta_{w} e_{w}^{*}+\eta_{f} e_{f}^{*}=E\right)$ the resulting equation is substantially "more" nonlinear than (31). It appears that its analytical solution is unavailable. We will solve the coupled constraint equilibrium problem (41) numerically using NIRA in Section 5.3. 


\subsection{A calibrated model}

Given the data in Table II, we would like to establish plausible values for the 6 parameters $\left(\alpha_{i}, \beta_{i}, \delta_{i}\right.$ for the two regions $\left.i=w, f\right)$ that characterise the regional revenue functions (39) (or (3)). The two functions (39) constitute two conditions that the parameters have to satisfy for a given value of price $p$.

We assume that the joint constraint (38) was not binding in 2000 i.e., in the year for which the data were collected in Table II. Since we claim that the regions are "game players", we have the following two first-order non-coupled-constraintequilibrium conditions

$$
\begin{aligned}
& e_{w}^{*}=\left(\frac{\beta_{w} \alpha_{w}}{p}\right)^{\frac{1}{1-\beta_{w}}}\left(e_{f}^{*}\right)^{\frac{\delta_{w}}{1-\beta_{w}}} \\
& e_{f}^{*}=\left(\frac{\beta_{f} \alpha_{f}}{p}\right)^{\frac{1}{1-\beta_{f}}}\left(e_{w}^{*}\right)^{\frac{\delta_{f}}{1-\beta_{f}}}
\end{aligned}
$$

which the coefficients also need to satisfy. Consequently, we have 4 equations in 6 variables.

Additionally, we have constraints on non-negativity of all parameters, $[0,1]$ membership of the exponents and that $\beta_{i}>\delta_{i}$. This means that we have notso-much freedom in choosing the parameters. A Matlab constrained minimisation function $^{19}$ was used to minimise the sum of deviations (squared, weighted) between the computed and historical revenues and between the postulated equilibrium energy consumptions and the historical consumptions. A solution (without any claim of uniqueness) is presented in Table III.

Table III: Parameters of regional revenue functions

\begin{tabular}{r||r|r} 
& Wallonia & Flanders \\
\hline$\alpha$ & 40592 & 43085 \\
\hline$\delta$ & 0.002359 & 0.025848 \\
\hline$\beta$ & 0.048545 & 0.037365
\end{tabular}

We conjecture that the parameter values in Table III, can represent a stylised regional competition problem. As said, we make no claim on any sort of uniqueness of theses parameters. (In particular a change of unites could diminish the values of $\alpha_{i}$ but we will stick to the "natural" units: Euro and boe.)

The calibrated game with the revenue functions (39) and constraint (38) is particular in several respects and so will be the numerical solutions to the game, presented below. For example, $\delta_{f}>\delta_{w}$ suggests that Flanders relies on the positive externality that Wallonia produces more than the other way around ${ }^{20}$; the inequality $\beta_{f}<\delta_{w}$ jointly with $\alpha_{f}>\alpha_{w}$ may reflect better efficiency of Flanders' use of energy, albeit for a finite range. In brief, we believe that the calibrated model used

\footnotetext{
${ }^{19}$ fmincon.

${ }^{20}$ Traditionally, Wallonia was a coal and steel producer. Perhaps $\delta_{f}>\delta_{w}$ captures Flanders' reliance on those products.
} 
in the rest of this paper enables us to analyse coupled constraint equilibria for a case study, which the model represents.

Notice that if $p=30 \mathrm{EURO} /$ boe the revenue functions (39) reproduce the statistical data displayed in Table II:

$$
\begin{aligned}
& 40592(70.51)^{0.002359} \cdot(82.18)^{0.048545}-30 \cdot 82.18=48332 \\
& 43085(82.18)^{0.025848} \cdot(70.51)^{0.037365}-30 \cdot 70.51=54493
\end{aligned}
$$

\subsection{Numerical solutions}

We have verified that the determinant of $\mathcal{H}_{2}>0$ for the adopted parameter values.

\subsubsection{Sharing the constraint's burden in solidarity or the "status quo" solutions}

We are interested to know how the regions respond to the imposition of the energy constraint (38). We will assume that the revenue functions' coefficients proposed in Table III do not depend on the energy use and solve the coupled constraint game (41) numerically for several values of $E$. We will keep the price $p$ constant for these experiments. $^{21}$

In this section we examine the regions' reaction to the imposition of the energy constraint under the assumption that they share the responsibility for the constraint's satisfaction in solidarity i.e., the weights are $r_{w}=r_{f}=1$. To see the scope for the regulator's interventions we will analyse this competitive solution against the symmetric Pareto-optimal ("efficient") solution.

In the figures that follow the solid lines correspond to the former while the dotted lines represent the latter. We will use an upper bar ${ }^{-}$to denote the Pareto optimal solutions; asterisk * will be used for coupled constraint equilibrium solutions.

Figure 1, scaled in EURO per-unit-of-labour, and Figure 2, in EURO, show how the regional and national revenues change when energy constraints are introduced. The horizontal axis represents the energy availability so, tightening of the constraint corresponds to "moving" from right to left.

The bottom panel in each figure corresponds to the value added of Wallonia, the middle one represents the Flanders' value and the top graph is the sum of both and is meant to describe Belgium's (total) national revenue ${ }^{22}$.

\footnotetext{
${ }^{21}$ In real life, a higher price of energy would cause the value added to increase. Our regional revenue model (39) does not allow for this effect so, the assumption of a constant price might correspond to the value added expressed in constant prices. Also, should the price increase substantially the constraint would not be binding.

${ }^{22}$ As said in Section 2.1 we neglect the contributions of Brussels.
} 

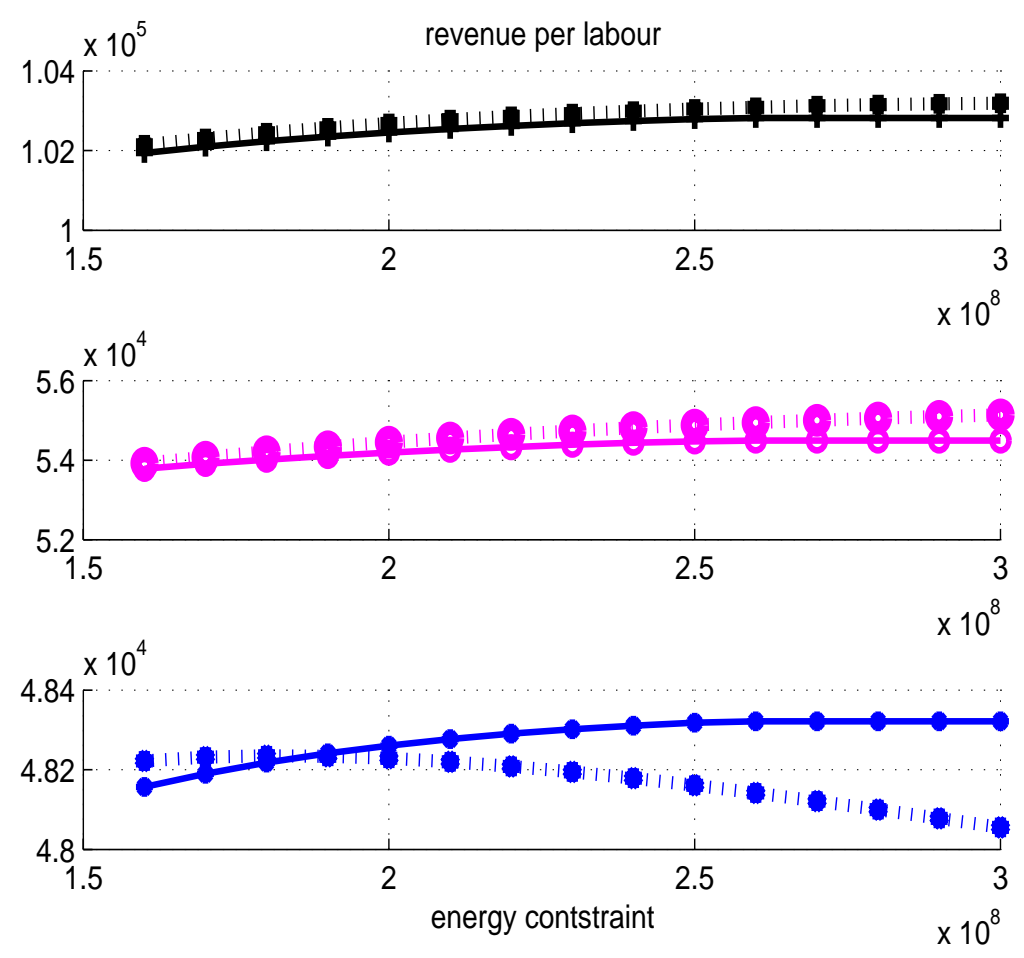

Figure 1: Revenue per unit of labour as a function of the energy constraints.
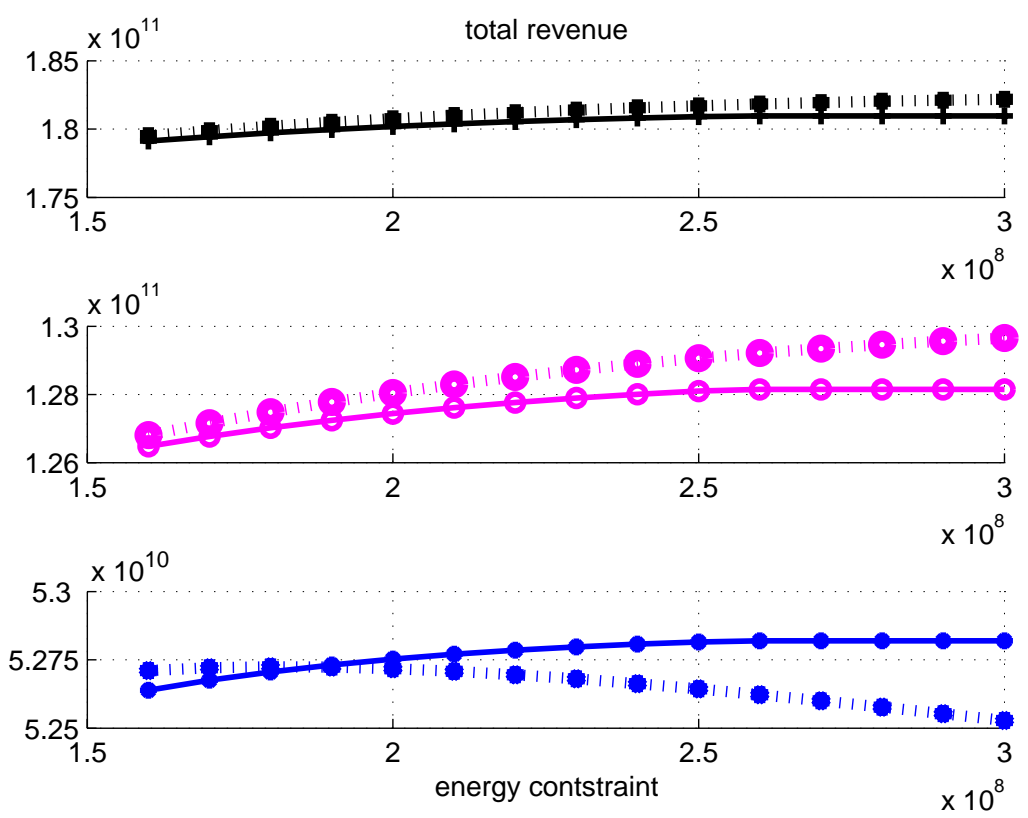

Figure 2: Total revenue as a function of the energy constraints. 


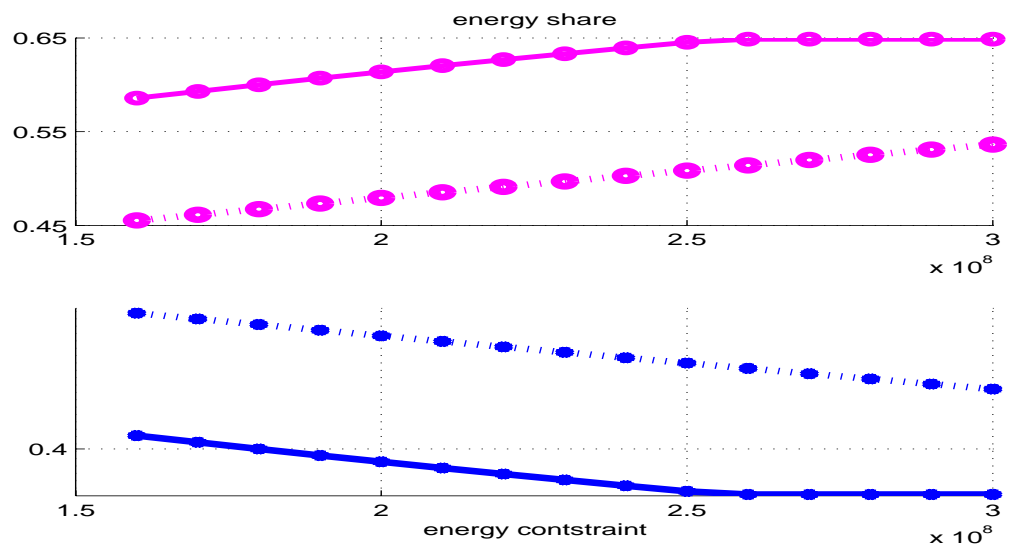

Figure 3: Wallonia's and Flanders' energy usage shares as functions of the energy constraints.

As expected, all revenues diminish if the available energy decreases. However, under the efficient solution, Wallonia is supposed to increase its contributions to the national revenue. This would be a result of assigning Wallonia a higher share of the energy consumption, as shown in Figure 3. (We notice that the constraint becomes active at $E=2.5 \cdot 10^{8}$ for the competitive solutions. The efficient solutions rely always on the whole energy quota, see Figure 5.)

A higher energy share apportioned to Wallonia might be "required" for the revenue maximisation because Wallonia's positive externality is "needed" for Flanders' output. Also, notice that under the Pareto optimal solution, Flanders' revenue per-unit-of-labour per-energy-input, see Figure 4, is growing faster than Wallonia's. This appears to compensate a possible decrease of Flander's output due to a more favourable treatment of Wallonia. 

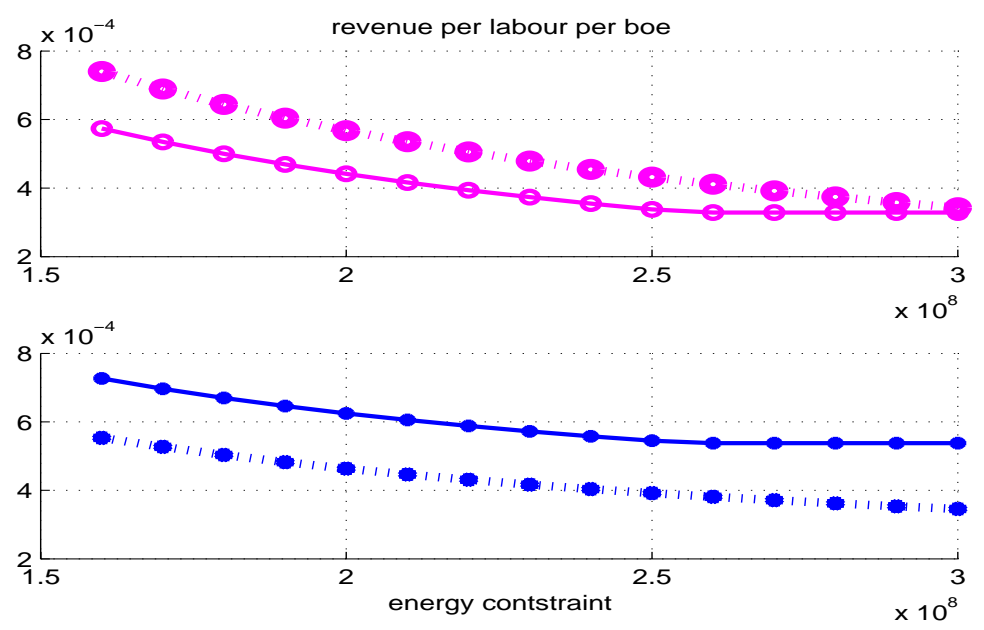

Figure 4: Wallonia's and Flanders' revenue generation efficiency as functions of the energy constraints.

It is interesting to notice that the competitive solutions are not only inefficient regarding the revenues they can generate but also in terms of the constraint's saturation. Figure 5 shows the constraint's slacks as the constraints are tightened. It is clear that the constraint is not binding for $E>250000000$ boe for the competitive solutions. Conversely, the regions always work to their "full capacity" if a Pareto optimal solution is implemented, see the dotted line at the level of zero.

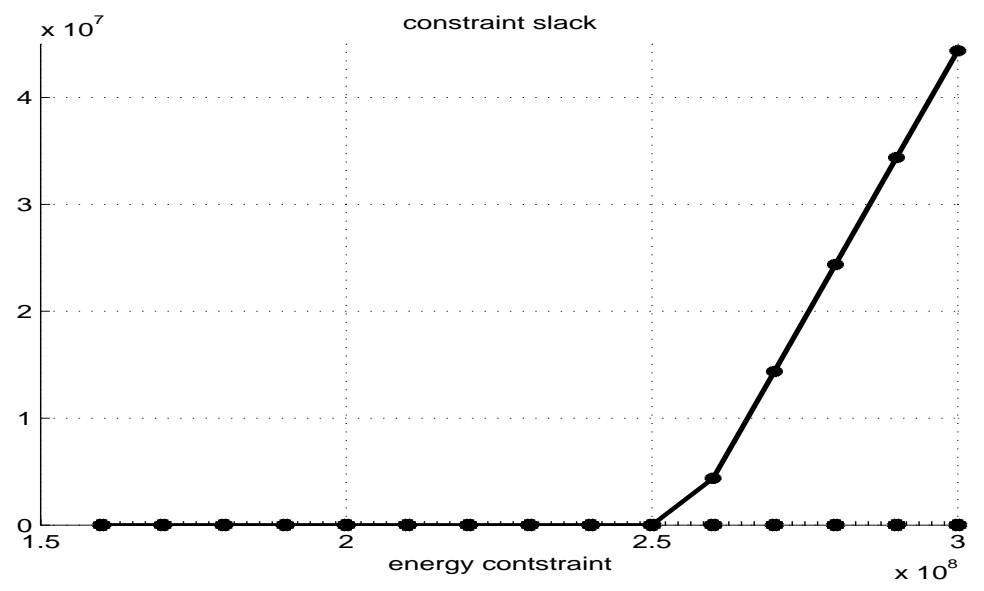

Figure 5: The constraint's slack as a function of tightening the constraints. 
Finally, it is interesting to compare the shadow prices for the competitive and efficient solutions. Figure 6 documents that higher prices are needed to support the Pareto symmetric optimal solution, than for a game equilibrium.
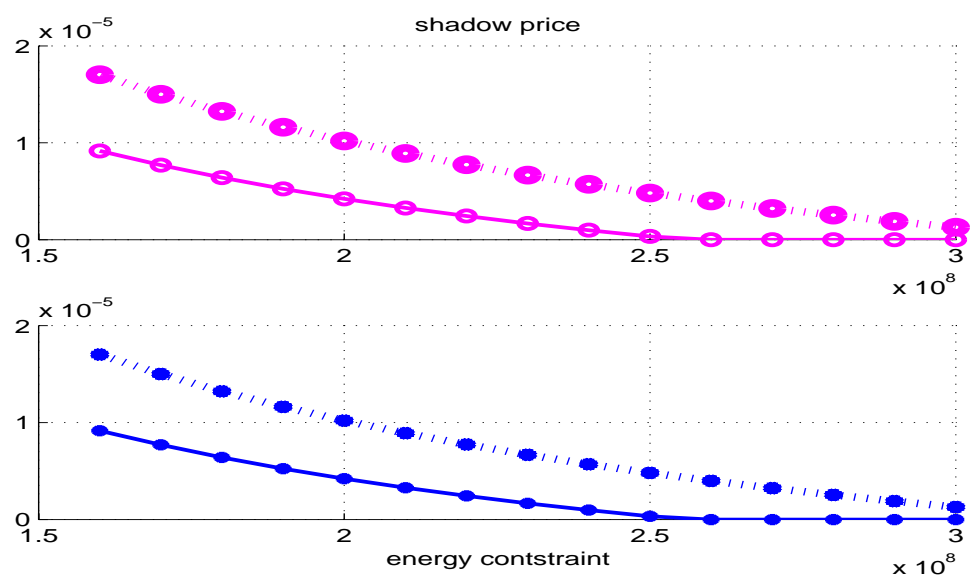

Figure 6: The shadow prices as functions of the energy constraints.

The observation about high shadow prices required for more socially acceptable outcomes will be exploited in the following section. By increasing one weight $\left(r_{w}\right.$ or $r_{f}$ ) the responsibility for the constraint's satisfaction will be lessen for one region. This will encourage this region to consume more energy. If this is the "right" region, the total revenue might increase in a new equilibrium.

We will see that an unequal weight $>1$ can produce a higher common-constraint shadow price and help the equilibrium become closer to the optimal solution.

\subsubsection{Asymmetrical sharing rules}

Here, we compute the regions' reactions to imposition of an energy constraint when the responsibility for the constraint's satisfaction is distributed unevenly. In particular, we will construct constrained equilibria when the marginal cost of violating the energy constraint for Flanders will be weighted by a series of $\frac{1}{r_{f}}$ as follows

$$
\frac{1}{r_{f}}:=3,2,1.5,1, \frac{3}{4}, \frac{1}{2}, \frac{1}{3} .
$$

Obviously, these weights correspond to

$$
r_{f}:=\frac{1}{3}, \frac{1}{2}, \frac{3}{4}, 1,1.5,2,3 .
$$

The other weight ${ }^{23}$ will be kept $r_{w}=1$.

Most of the results in this section will be presented in three-dimensional spaces where the first dimension is $r_{f} \in\left\{\frac{1}{3}, \frac{1}{2}, \frac{3}{4}, 1,1.5,2,3\right\}$ and the second dimension is

\footnotetext{
${ }^{23}$ See footnote 16 and Appendix B.
} 
the available energy $E \in[1.5,3] \times 10^{8}$ boe. The variable of interest will be presented in the third dimension.

Inspired by the observation that the shadow price for the symmetric Pareto optimal solution dominates the equilibrium shadow price (see Figure 6) we will examine whether varying $r_{f}$ can indeed generate $\lambda^{*}$ that would resemble $\bar{\lambda}$.

We first show how the Pareto solution shadow price can be represented in $3 \mathrm{D}$, see Figure 7. In essence, this is the top line in Figure 6 (either panel) shown as a surface where all iso-lines are parallel to $r_{f}$ (first dimension).

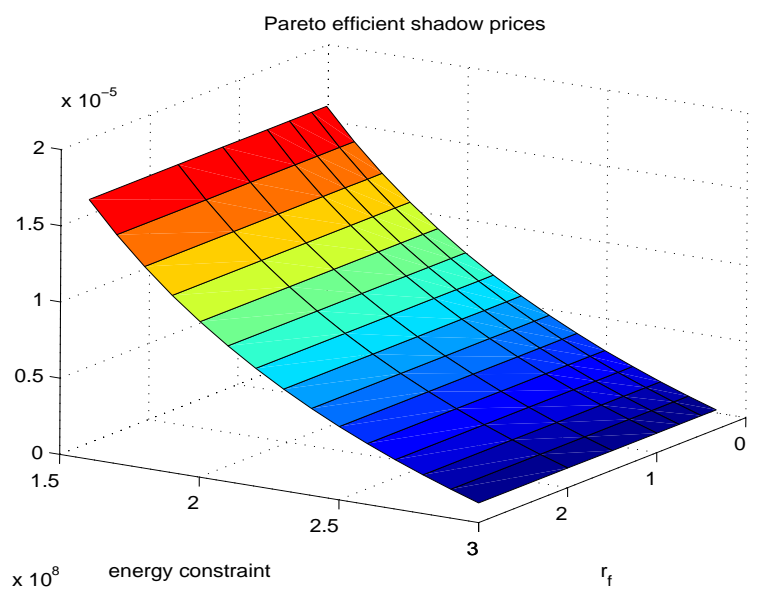

Figure 7: The symmetric Pareto optimal shadow prices in 3D.

This surface could be added as the "ceiling" to the following 3D graphs in Figure 8 .
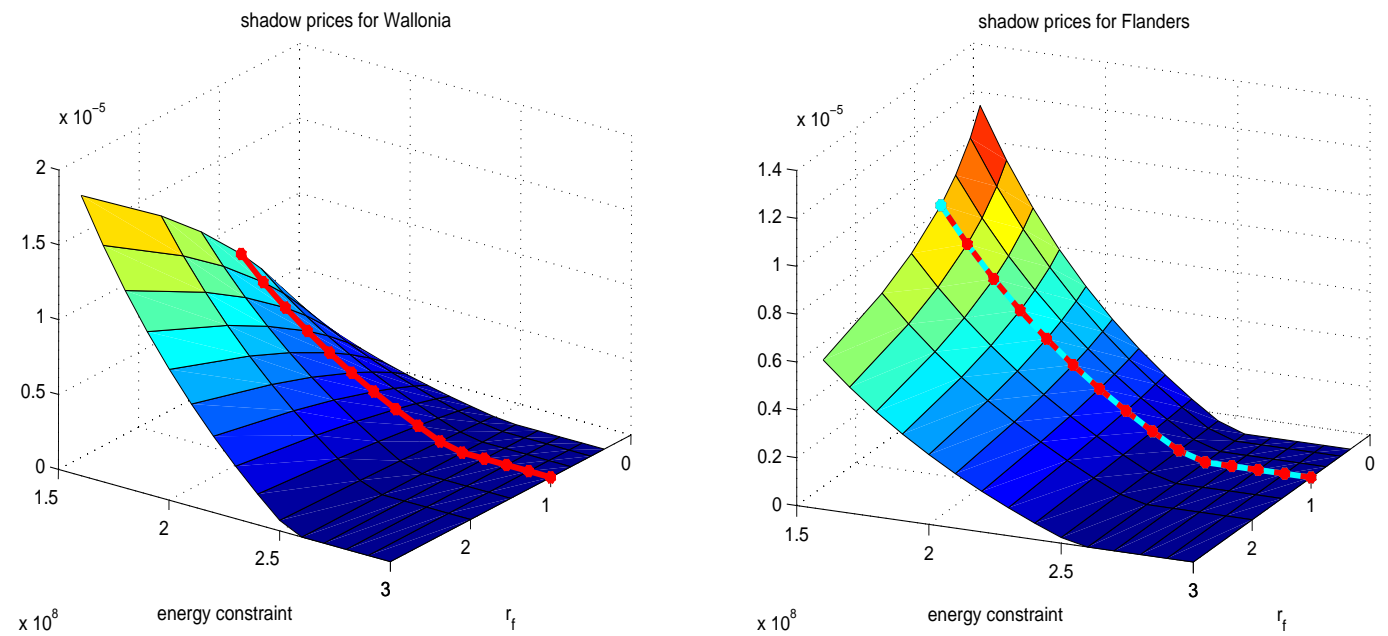

Figure 8: The equilibrium shadow prices as functions of the weight $r_{f}$ and the energy constraints. 
However, we will not do it to not "cover" the commented graph. ${ }^{24}$ What we show in this figure is how the equilibrium shadow prices $\lambda^{*}$ change in $r_{f}$, and in $E$, for Wallonia (left panel) and Flanders (right panel). The red dotted lines represent the symmetric equilibrium shadow prices (i.e., $r_{f}=1$ as in Figure 6).

The shadow price for Flanders is $\frac{\lambda^{*}}{r_{f}}$ while for Wallonia it is just $\lambda^{*}$. We can clearly see that $\lambda^{*}$ increases as $r_{f}$ rises. A comparison between the values reached by $\bar{\lambda}$ in Figure 7 and $\lambda^{*}$ in Figure 8 (left panel) suggests that varying $r_{f}$ might diminish the difference $\bar{\lambda}-\lambda^{*}$. Consequently, some equilibria might be socially more desirable than some other equilibria. We will verify this conjecture by examining the total revenue for the country as a function of $r_{f}$ (see Figure 9).

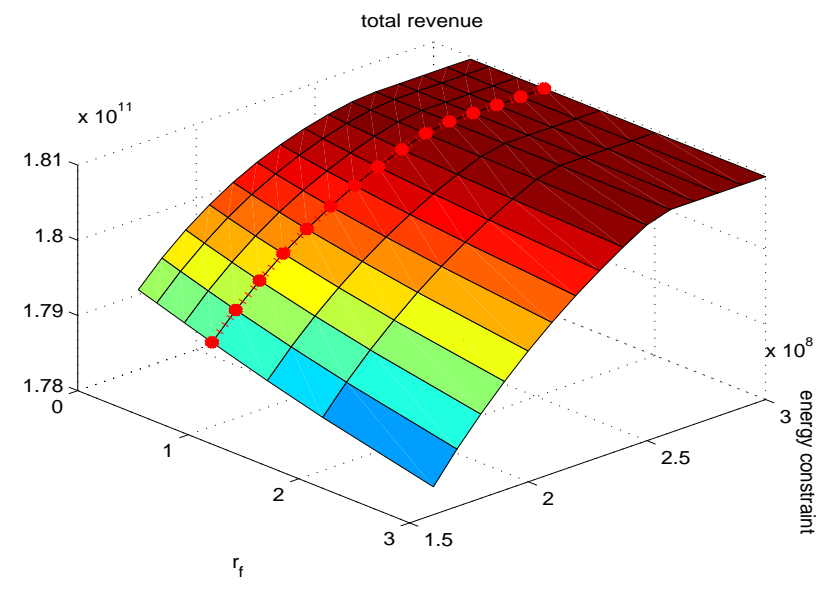

Figure 9: Total revenue of the country as a function of the weight $r_{f}$ and the energy constraints.

We can see in Figure 9 that the total revenue increases in $\frac{1}{r_{f}}$. This corresponds to shifting the responsibility for the constraint's satisfaction away from Wallonia and allowing it to consume more energy.

In the context of higher energy efficiency of Flanders vis-a-vis Wallonia this might be a surprising conclusion. Let us examine what outcomes are caused by the variation of $r_{f}$ at the regional level.

Figure 10 shows the regional revenues' dependence on $r_{f}$ and $E$. We observe that the preferential treatment of Wallonia ( small $r_{f}$ ) suits both regions well, albeit Wallonia appears to gain more.

\footnotetext{
${ }^{24}$ We could produce the Pareto efficient "ceilings" for all the remaining figures. However, this could blur the analysis. Instead, we will make occasional references to the respective Pareto solutions represented by the dotted lines in Figures 2- 6 .
} 

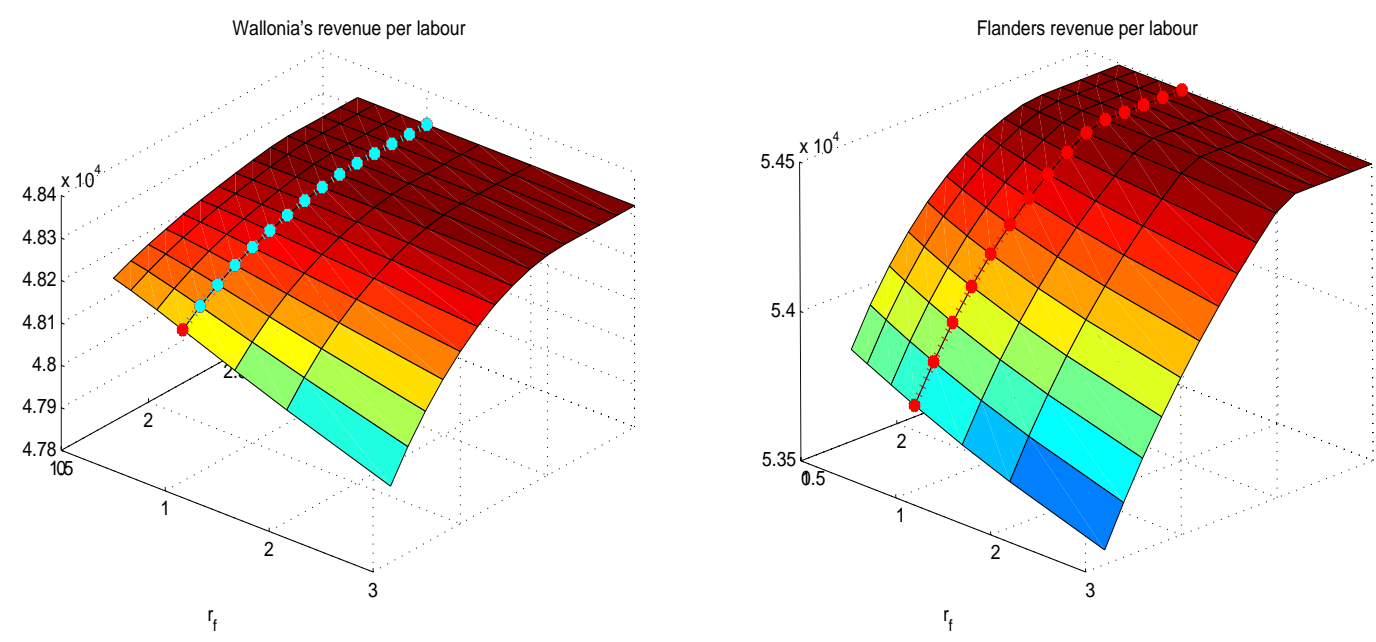

Figure 10: The regional revenues per unit of labour as functions of the weight $r_{f}$ and the energy constraints.

Of great interest is to examine the regional equilibrium strategies, which lead to the above revenue outcomes. Figure 11 shows the strategic decisions of how much energy should be consumed per unit of labour in regions.
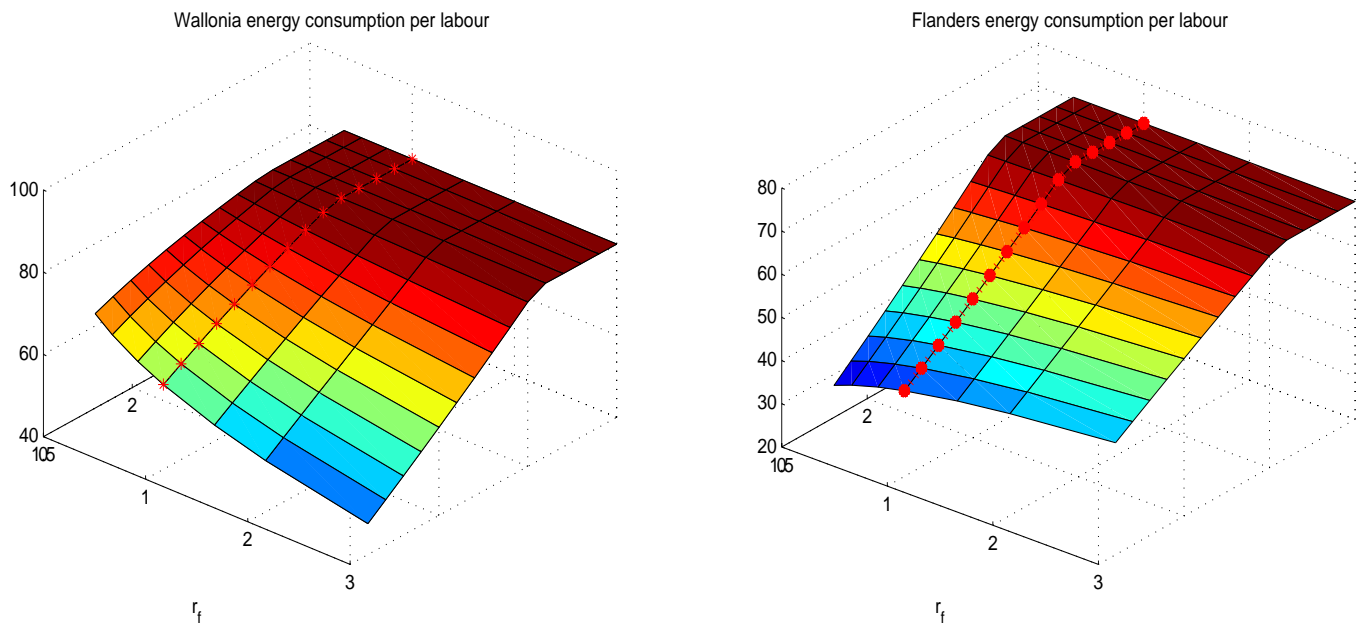

Figure 11: The regional strategies boe/labour as functions of the weight $r_{f}$ and the energy constraints.

Here we can see that increasing weight $r_{f}$ encourages Flanders to use more energy while lowering it pushes Wallonia to consume more. These tendencies are even more visible when we observe the energy consumption shares in Figure 12. 

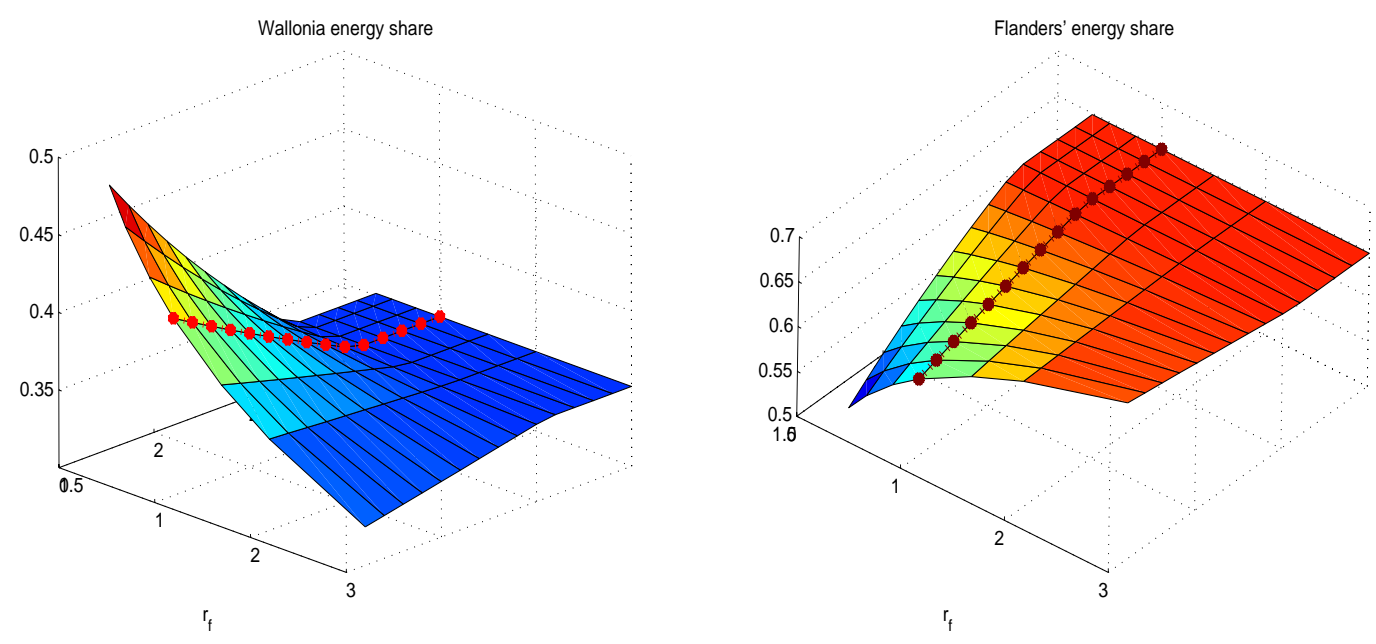

Figure 12: The regional energy consumption shares as functions of the weight $r_{f}$ and the energy constraints.

Finally we can remark that the coupled constrained equilibria are "efficient" in that the slack on the constraint is zero, see Figure 13.

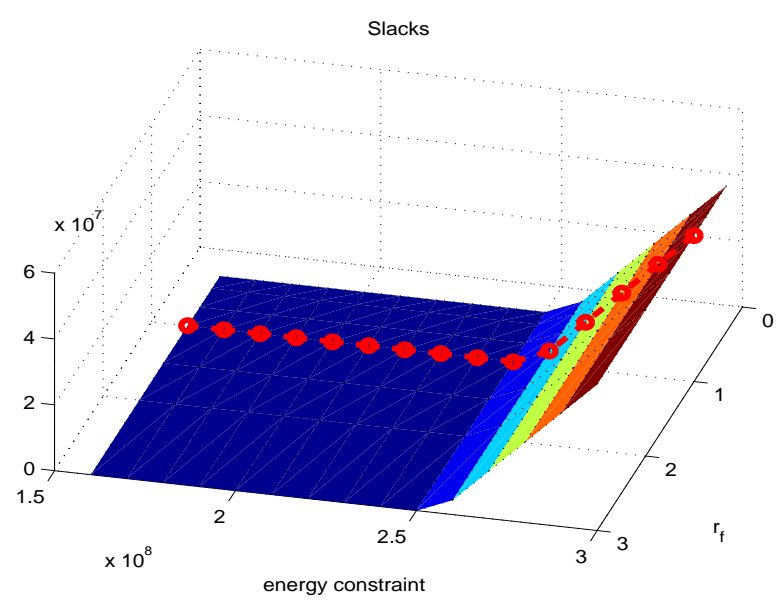

Figure 13: Total revenue of the country as a function of the weight $r_{f}$ and the energy constraints.

\subsubsection{A variation in the reliance on externality}

In our model, the existence of inter-regional externalities is a fundamental ingredient of the problem: depending on the size of the positive externality exerted by the energy-less-efficient region on the more efficient one, the latter should be apportioned the higher or the lower energy share.

Compare the following figures, obtained for a decreased reliance of Flanders on the positive externality produced by Wallonia $\left(\delta_{f}=.002\right)$, with Figures 9 and 10 . 


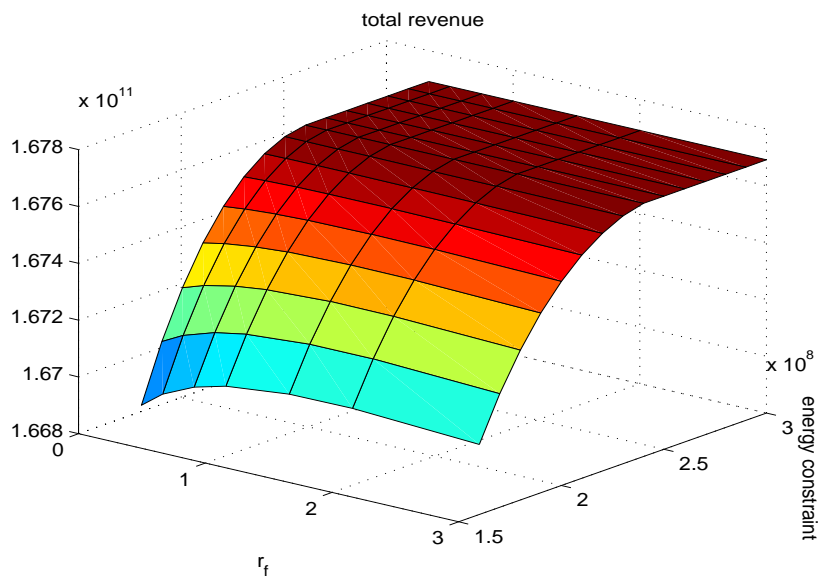

Figure 14: Total revenue of the country as a function of the weight $r_{f}$ and the energy constraints.
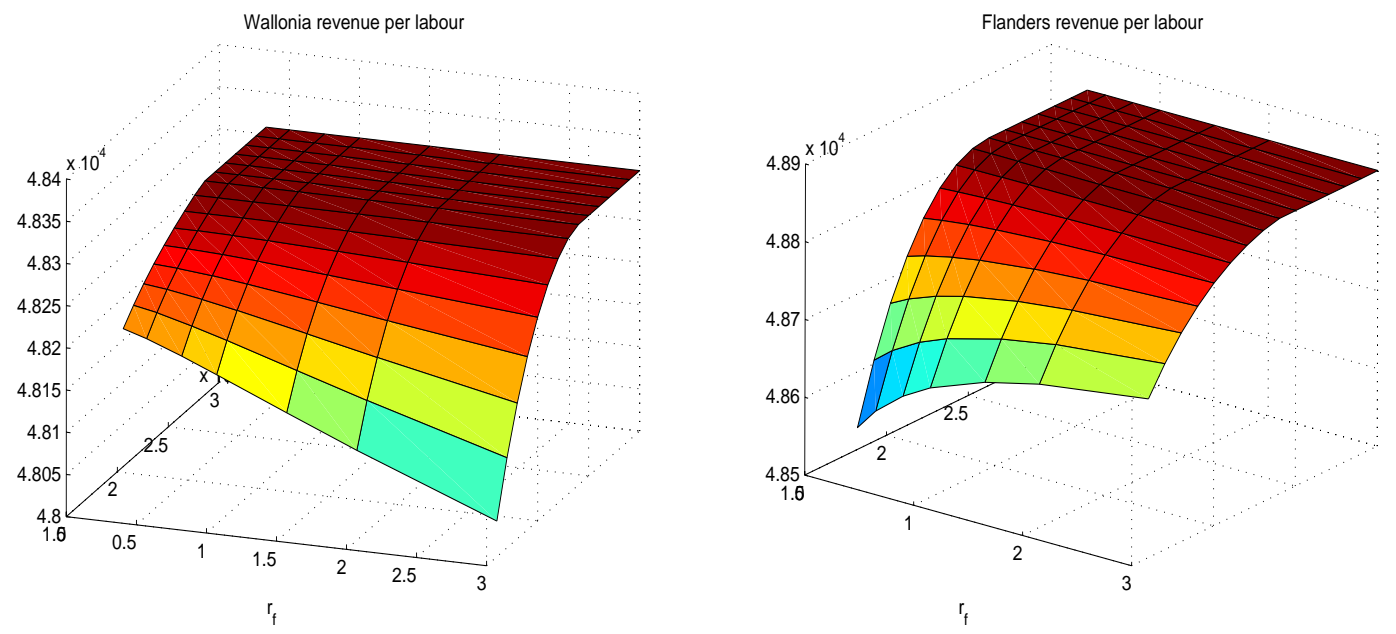

Figure 15: The regional revenues per unit of labour as functions of the weight $r_{f}$ and the energy constraints. 
We can clearly see that, now, a preferential treatment of Flanders (i.e., $r_{f}$ increases) results in higher energy consumption by this region. However, the overall country's revenue growth appears non monotonic in $r_{f}$.

\section{Concluding remarks}

We have proposed a novel methodological approach to regional cost sharing of environmental regulation. The framework is game theoretic, based on the concept of coupled constraint equilibrium, allowing us to formulate naturally an important policy problem of national governments of multi-regional countries. The problem is timely and concerns the implementation of international agreements like the Kyoto Protocol. In particular, there are two specific questions that our model helps answer: how to efficiently share the burden of environment regulations (like emissions quotas) across regions? And, how to enforce such a sharing?

The problem is particularly acute when there exist significant structural differences across regions. In the case considered in our paper, regions may differ in their energy efficiency. For example, a region may be (for many good reasons) much more energy intensive than another region(s). If the national government has to allocate emission permits across the regions, what could be the most efficient sharing rule for the country?

In order to give substance to this discussion, we have considered the case Wallonia vs. Flanders. Wallonia is traditionally significantly more energy intensive than Flanders while the contribution of the latter to Belgian GDP is clearly larger.

It could be thought that having to enforce a national pollution norm, in accordance with international agreements, the regulator should penalise the more polluting, or deviating, region, especially if its contribution to national wealth is markedly lower than that of the less polluting regions. This is clearly the case of Wallonia in Belgium. Our paper makes a point in this respect: the reasoning that leads to limiting Wallonia's energy use, does not take into account the fact that regions do interact in several meaningful ways such that penalising the more deviating region (from an energy-efficiency norm) may turn out to be inefficient in terms of the joint production maximisation. In our model, the existence of interregional externalities is a fundamental ingredient of the story. We surmise that the decision of apportioning the higher, or the lower, energy share to the more efficient, or disciplined, region must depend on the size of the positive externality exerted by the more deviating region on the former.

Hence, there is no simple theorem for efficient regulation of cost sharing across regions. One has not only to look at the differences in factor intensity but also to scrutinise the economic interactions between regions, which is far from easy. Even if one restricts these interactions to inter-regional technological spillovers, the issue is not so simple since a substantial part of these spillovers is intangible. Our analysis points at a further and more political ingredient: the government may choose an uneven distribution (across regions) of the responsibility for the joint constraint satisfaction to force a particular outcome. Our paper shows clearly that the shape of equilibria identified and the corresponding national revenues tightly depend on the parameter $r_{f}$. This opens a further important line of research: what could be 
an optimal ${ }^{25} r_{f}$ ? Our numerical analysis sheds light on some particular properties of our model in this respect. Our ambition is to provide a more general appraisal in less specific models regarding this question, which seems to us crucial in the design of environmental regulation policies.

\section{Acknowledgment}

The authors extend their thanks to Marc Germain for comments on this paper and to Nathaniel Robson whose assistance in the completion of this paper was supported by a Victoria University of Wellington URF grant 32665/Award-2155.

\section{References}

[1] The National Bank of Belgium Online Database, http://www.nbb.be/pub/05_00_00_00_00/05_01_01_00_00.htm?t=ho\&l=en, available on 5 June 2008.

[2] S. Berridge and J. B. Krawczyk, Relaxation algorithms in finding Nash equilibria, Economic Working Papers Archive, 1997, URL: http://econwpa.wustl.edu/eprints/comp/papers/9707/9707002.abs.

[3] R. Boucekkine and M. Germain, The burden sharing of pollution abatement costs in multi-regional open economies, CORE Discussion Paper 2007-11, 2007.

[4] J. Contreras, M. Klusch, and J. B. Krawczyk, Numerical solutions to NashCournot equilibria in coupled constraint electricity markets, IEEE Transactions on Power Systems 19 (2004), no. 1, 195-206.

[5] J. Contreras, J. B. Krawczyk, and J. Zuccollo, Electricity market games with constraints on transmission capacity and emissions, 30th Conference of the International Association for Energy Economics, February 2007, Wellington, New Zealand.

[6] D. Devogelaer, Gusbin; D., and Janssen L., Régionalisation des perspectives énergétiques pour la Belgique à l'horizon 2030: résultats pour la Région de Bruxelles-Capitale, Working Paper, 9-07, Brueau Fédéral du Plan, 2007.

[7] L. Drouet, A. Haurie, F. Moresino, J.-P. Vial, M. Vielle, and L. Viguier, An oracle based method to compute a coupled equilibrium in a model of international climate policy, Computational Management Science DOI 10.100\%/s10287-00\%0043-2 (2007), in print.

[8] M. Germain, Ph. Monfort, and Th. Bréchet, Allocation des efforts de dépollution dans des économies avec spécialisation internationale, Revue Economique 57 (2006), no. 2, 219-239.

\footnotetext{
${ }^{25}$ In "real-life", the government might add other criteria to maximisation of the current total revenue when choosing a value of $r_{f}$. For example, $r_{f}$ may need to be greater than one resulting from Figures 2 and 9, if the government wanted region $w$ to restructure.
} 
[9] A. Haurie, Environmental coordination in dynamic oligopolistic markets, Group Decision and Negotiation 4 (1994), 46-67.

[10] A. Haurie and J. B. Krawczyk, Optimal charges on river effluent from lumped and distributed sources, Environmental Modelling and Assessment 2 (1997), 177-199.

[11] B. E. Hobbs and J.-S. Pang, Nash-Cournot equilibria in electric power markets with piecewise linear demand functions and joint constraints, Operations Research 55 (2007), no. 1, 113-127.

[12] J. B. Krawczyk, Coupled constraint Nash equilibria in environmental games, Resource and Energy Economics 27 (2005), 157-181.

[13] _ Numerical solutions to coupled-constraint (or generalised) Nash equilibrium problems, Computational Management Science (Online Date: November 09, 2006), http://dx.doi.org/10.1007/s10287-006-0033-9.

[14] J. B. Krawczyk, O. Pourtallier, and M. Tidball, A steady-state satisfactory solution to an environmental game with piece-wise defined payoffs, Natural Resource and Modeling 11 (1998), no. 4, 301-329.

[15] J. B. Krawczyk and S. Uryasev, Relaxation algorithms to find Nash equilibria with economic applications, Environmental Modelling and Assessment $\mathbf{5}$ (2000), 63-73.

[16] J. B. Krawczyk and J. Zuccollo, NIRA-3: A MATLAB package for finding Nash equilibria in infinite games, Working Paper, School of Economics and Finance, VUW, 2006.

[17] E. A. Nurminski, Progress in nondifferentiable optimization, ch. Subgradient Method for Minimizing Weakly Convex Functions and $\epsilon$-Subgradient Methods of Convex Optimisation, pp. 97-123, International Institute for Applied Systems Analysis, Laxenburg, Austria, 1982.

[18] J.-S. Pang and M. Fukushima, Quasi-variational inequalities, generalized Nash equilibria and multi-leader-follower games, Computational Management Science 1 (2005), 21-56.

[19] P. M. Romer, Increasing returns and long-run growth, Journal of Political Economy 94 (1986), 1002-1037.

[20] J. B. Rosen, Existence and uniqueness of equilibrium points for concave nperson games, Econometrica 33 (1965), no. 3, 520-534.

[21] S Uryasev, Adaptive Algorithms for Stochastic Optimisation and Game Theory, Nauka, Moscow, 1990.

[22] S. Uryasev and R. Y. Rubinstein, On relaxation algorithms in computation of noncooperative equilibria, IEEE Transactions on Automatic Control 39 (1994), no. $6,1263-1267$. 


\section{Appendix}

\section{A NIRA}

\section{A.1 The Nikaido-Isoda function}

This function is a cornerstone of the NIRA technique for solving games for their CCE. It transforms the complex process of solving a (constrained) game into a far simpler (constrained) optimisation problem.

Definition A.1. Let $\Pi_{i}$ be the payoff function for player $f, X$ a collective strategy set as before and $r_{i}>0$ be a given weighting ${ }^{26}$ of player $f$. The Nikaido-Isoda function $\Psi: X \times X \rightarrow \mathbb{R}$ is defined as

$$
\Psi(\mathbf{x}, \mathbf{y})=\sum_{f=1}^{F} r_{i}\left[\Pi_{i}\left(y_{i} \mid \mathbf{x}\right)-\Pi_{i}(\mathbf{x})\right]
$$

Result A.1. See [22].

$$
\Psi(\mathbf{x}, \mathbf{x}) \equiv 0 \quad \mathbf{x} \in X
$$

Each summand from the Nikaido-Isoda function can be thought of as the improvement in payoff a player will receive by changing his action from $x_{f}$ to $y_{f}$ while all other players continue to play according to $\mathbf{x}$. Therefore, the function represents the sum of these improvements in payoff. Note that the maximum value this function can take, for a given $\mathbf{x}$, is always nonnegative, owing to Result A.1 above. The function is everywhere non-positive when either $\mathbf{x}$ or $\mathbf{y}$ is a Nash equilibrium point, since in an equilibrium situation no player can make any improvement to their payoff. Consequently, each summand in this case can be at most zero at the Nash equilibrium point [15].

We observe that the "sum of improvements" in $\Psi$ depends on the weighting vector $\mathbf{r}=\left(r_{i}\right)_{f \in F}$. Consequently, a manifold of equilibria indexed by $\mathbf{r}$ is expected to exist. However, for a given $\mathbf{r}$ and diagonal strict concavity of $\sum_{f \in F} r_{i} \Pi_{i}\left(x_{i}\right)$, uniqueness of equilibrium $\mathbf{x}^{*}$ is guaranteed, see [20] and [10]. Also, notice that according to Theorem 3.2 (the convergence theorem), if the assumptions are fulfilled, then NIRA converges to the unique equilibrium, for the value of $\mathbf{r}$ that was used in the definition of $\Psi$.

When the Nikaido-Isoda function cannot be made (significantly) positive for a given $\mathbf{y}$, we have (approximately) reached the Nash equilibrium point. This observation is used to construct a termination condition for the relaxation algorithm, which is used to min-maximise $\Psi$. An $\varepsilon$ is chosen such that, when

$$
\max _{\mathbf{y} \in \mathbb{R}^{m}} \Psi\left(\mathbf{x}^{s}, \mathbf{y}\right)<\varepsilon
$$

\footnotetext{
${ }^{26}$ The weights can be viewed as a political instrument the regulator might use to distribute the responsibility for the joint constraints' satisfaction, among the generators (and periods).
} 
(where $\mathbf{x}^{s}$ is the $s$-th iteration approximation of $x^{*}$ ) the Nash equilibrium would be achieved to a sufficient degree of precision [15].

The Nikaido-Isoda function is used to construct the optimum response function. This function is similar to the best response function in standard non-cooperative game theory. It defines each player's optimal action to maximise his payoff given what the other players have chosen. The vector $Z(\mathbf{x})$ gives the 'best move' of each player when faced with the collective action $\mathbf{x}$. It is at this point that the coupled constraints are introduced into the optimisation problem. The maximisation of the Nikaido-Isoda function in equation (49) is performed subject to the constraints on the players' actions.

Definition A.2. The optimum response function at point $\mathbf{x}$ is

$$
Z(\mathbf{x}) \in \arg \max _{\mathbf{y} \in X} \Psi(\mathbf{x}, \mathbf{y})
$$

\section{A.2 The relaxation algorithm}

The relaxation algorithm iterates the function $\Psi$ to find the Nash equilibrium of a game. It starts with an initial estimate of the Nash equilibrium and iterates from that point towards $Z(\mathbf{x})$ until no more improvement is possible. At such a point every player is playing their optimum response to every other player's action and the Nash equilibrium is reached. The relaxation algorithm, when $Z(\mathbf{x})$ is single-valued, is

$$
\begin{array}{ll}
\mathbf{x}^{s+1}=\left(1-\alpha_{s}\right) \mathbf{x}^{s}+\alpha_{s} Z\left(\mathbf{x}^{s}\right) \quad & 0<\alpha_{s} \leq 1 \\
& s=0,1,2, \ldots
\end{array}
$$

From the initial estimate, an iterate step $s+1$ is constructed by a weighted average of the players' improvement point $Z\left(\mathbf{x}^{s}\right)$ and the current action point $\mathbf{x}^{s}$. Given concavity assumptions explained in section 3.2, this averaging ensures convergence (see [22], [15]) to the Nash equilibrium by the algorithm. By taking a sufficient number of iterations of the algorithm, the Nash equilibrium $\mathbf{x}^{*}$ can be determined with a specified precision.

\section{B Rosen's weights in $\mathbb{R}_{+}^{2}$}

Consider a game with payoffs $\Pi_{1}(e), \Pi_{2}(e)$ that satisfy $(12)$ (diagonal strict concavity). So, we know that this game has a unique equilibrium for a choice of $r_{1}, r_{2}$. The equilibrium fist order conditions are

$$
\left.\begin{array}{c}
\frac{\partial \Pi_{1}(e)}{\partial e_{1}}=-\frac{\lambda\left(r_{1}, r_{2}\right)}{r_{1}} \\
\frac{\partial \Pi_{2}(e)}{\partial e_{2}}=-\frac{\lambda\left(r_{1}, r_{2}\right)}{r_{2}}
\end{array}\right\}
$$


where $\lambda \geq 0$ is the shadow price of the common constraint of type (7). We notice that conditions (51) are equivalent to

$$
\left.\begin{array}{l}
\frac{\partial \Pi_{1}(e)}{\partial e_{1}}=-\frac{\lambda(r, 1)}{r} \\
\frac{\partial \Pi_{2}(e)}{\partial e_{2}}=-\lambda(r, 1)
\end{array}\right\}
$$

if

$$
\left.\begin{array}{l}
\frac{\lambda\left(r_{1}, r_{2}\right)}{r_{1}}=\frac{\lambda^{\prime}(r, 1)}{r} \\
\frac{\lambda\left(r_{1}, r_{2}\right)}{r_{2}}=\lambda^{\prime}(r, 1)
\end{array}\right\} .
$$

The above is true if

$$
r \equiv \frac{r_{1}}{r_{2}}
$$




\section{Recent titles \\ CORE Discussion Papers}

2008/18. Juan D. MORENO-TERNERO and John E. ROEMER. Axiomatic resource allocation for heterogeneous agents.

2008/19. Carlo CAPUANO and Giuseppe DE FEO. Mixed duopoly, privatization and the shadow cost of public funds.

2008/20. Helmuth CREMER, Philippe DE DONDER, Dario MALDONADO and Pierre PESTIEAU. Forced saving, redistribution and nonlinear social security schemes.

2008/21. Philippe CHEVALIER and Jean-Christophe VAN DEN SCHRIECK. Approximating multiple class queueing models with loss models.

2008/22. Pierre PESTIEAU and Uri M. POSSEN. Interaction of defined benefit pension plans and social security.

2008/23. Marco MARINUCCI. Optimal ownership in joint ventures with contributions of asymmetric partners.

2008/24. Raouf BOUCEKKINE, Natali HRITONENKO and Yuri YATSENKO. Optimal firm behavior under environmental constraints.

2008/25. Ana MAULEON, Vincent VANNETELBOSCH and Cecilia VERGARI. Market integration in network industries.

2008/26. Leonidas C. KOUTSOUGERAS and Nicholas ZIROS. Decentralization of the core through Nash equilibrium.

2008/27. Jean J. GABSZEWICZ, Didier LAUSSEL and Ornella TAROLA. To acquire, or to compete? An entry dilemma.

2008/28. Jean-Sébastien TRANCREZ, Philippe CHEVALIER and Pierre SEMAL. Probability masses fitting in the analysis of manufacturing flow lines.

2008/29. Marie-Louise LEROUX. Endogenous differential mortality, non monitored effort and optimal non linear taxation.

2008/30. Santanu S. DEY and Laurence A. WOLSEY. Two row mixed integer cuts via lifting.

2008/31. Helmuth CREMER, Philippe DE DONDER, Dario MALDONADO and Pierre PESTIEAU. Taxing sin goods and subsidizing health care.

2008/32. Jean J. GABSZEWICZ, Didier LAUSSEL and Nathalie SONNAC. The TV news scheduling game when the newscaster's face matters.

2008/33. Didier LAUSSEL and Joana RESENDE. Does the absence of competition in the market foster competition for the market? A dynamic approach to aftermarkets.

2008/34. Vincent D. BLONDEL and Yurii NESTEROV. Polynomial-time computation of the joint spectral radius for some sets of nonnegative matrices.

2008/35. David DE LA CROIX and Clara DELAVALLADE. Democracy, rule of law, corruption incentives and growth.

2008/36. Jean J. GABSZEWICZ and Joana RESENDE. Uncertain quality, product variety and price competition. 2008/37. Gregor ZOETTL. On investment decisions in liberalized electricity markets: the impact of price caps at the spot market.

2008/38. Helmuth CREMER, Philippe DE DONDER, Dario MALDONADO and Pierre PESTIEAU. Habit formation and labor supply.

2008/39. Marie-Louise LEROUX and Grégory PONTHIERE. Optimal tax policy and expected longevity: a mean and variance approach.

2008/40. Kristian BEHRENS and Pierre M. PICARD. Transportation, freight rates, and economic geography.

2008/41. Gregor ZOETTL. Investment decisions in liberalized electricity markets: A framework of peak load pricing with strategic firms.

2008/42. Raouf BOUCEKKINE, Rodolphe DESBORDES and Hélène LATZER. How do epidemics induce behavioral changes?

2008/43. David DE LA CROIX and Marie VANDER DONCKT. Would empowering women initiate the demographic transition in least-developed countries? 


\section{Recent titles}

\section{CORE Discussion Papers - continued}

2008/44. Geoffrey CARUSO, Dominique PEETERS, Jean CAVAILHES and Mark ROUNSEVELL. Space-time patterns of urban sprawl, a 1D cellular automata and microeconomic approach.

2008/45. Taoufik BOUEZMARNI, Jeroen V.K. ROMBOUTS and Abderrahim TAAMOUTI. Asymptotic properties of the Bernstein density copula for dependent data.

2008/46. Joe THARAKAN and Jean-Philippe TROPEANO. On the impact of labor market matching on regional disparities.

2008/47. Shin-Huei WANG and Cheng HSIAO. An easy test for two stationary long processes being uncorrelated via AR approximations.

2008/48. David DE LA CROIX. Adult longevity and economic take-off: from Malthus to Ben-Porath.

2008/49. David DE LA CROIX and Gregory PONTHIERE. On the Golden Rule of capital accumulation under endogenous longevity.

2008/50. Jean J. GABSZEWICZ and Skerdilajda ZANAJ. Successive oligopolies and decreasing returns.

2008/51. Marie-Louise LEROUX, Pierre PESTIEAU and Grégory PONTHIERE. Optimal linear taxation under endogenous longevity.

2008/52. Yuri YATSENKO, Raouf BOUCEKKINE and Natali HRITONENKO. Estimating the dynamics of R\&D-based growth models.

2008/53. Roland Iwan LUTTENS and Marie-Anne VALFORT. Voting for redistribution under desertsensitive altruism.

2008/54. Sergei PEKARSKI. Budget deficits and inflation feedback.

2008/55. Raouf BOUCEKKINE, Jacek B. KRAWCZYK and Thomas VALLEE. Towards an understanding of tradeoffs between regional wealth, tightness of a common environmental constraint and the sharing rules.

\section{Books}

Y. POCHET and L. WOLSEY (eds.) (2006), Production planning by mixed integer programming. New York, Springer-Verlag.

P. PESTIEAU (ed.) (2006), The welfare state in the European Union: economic and social perspectives. Oxford, Oxford University Press.

H. TULKENS (ed.) (2006), Public goods, environmental externalities and fiscal competition. New York, Springer-Verlag.

V. GINSBURGH and D. THROSBY (eds.) (2006), Handbook of the economics of art and culture. Amsterdam, Elsevier.

J. GABSZEWICZ (ed.) (2006), La différenciation des produits. Paris, La découverte.

L. BAUWENS, W. POHLMEIER and D. VEREDAS (eds.) (2008), High frequency financial econometrics: recent developments. Heidelberg, Physica-Verlag.

P. VAN HENTENRYCKE and L. WOLSEY (eds.) (2007), Integration of AI and OR techniques in constraint programming for combinatorial optimization problems. Berlin, Springer.

\section{CORE Lecture Series}

C. GOURIÉROUX and A. MONFORT (1995), Simulation Based Econometric Methods.

A. RUBINSTEIN (1996), Lectures on Modeling Bounded Rationality.

J. RENEGAR (1999), A Mathematical View of Interior-Point Methods in Convex Optimization.

B.D. BERNHEIM and M.D. WHINSTON (1999), Anticompetitive Exclusion and Foreclosure Through Vertical Agreements.

D. BIENSTOCK (2001), Potential function methods for approximately solving linear programming problems: theory and practice.

R. AMIR (2002), Supermodularity and complementarity in economics.

R. WEISMANTEL (2006), Lectures on mixed nonlinear programming. 